\title{
Nonadditive entropy and nonextensive statistical mechanics - An overview after 20 years
}

\author{
Constantino Tsallis \\ Centro Brasileiro de Pesquisas Físicas and National Institute of Science and Technology for Complex Systems \\ Rua Xavier Sigaud 150, 22290-180 Rio de Janeiro, Brazil \\ and \\ Santa Fe Institute \\ 1399 Hyde Park Road, Santa Fe, NM 87501, USA
}

(Received on 17 April, 2009)

\begin{abstract}
Statistical mechanics constitutes one of the pillars of contemporary physics. Recognized as such — together with mechanics (classical, quantum, relativistic), electromagnetism and thermodynamics - , it is one of the mandatory theories studied at virtually all the intermediate- and advanced-level courses of physics around the world. As it normally happens with such basic scientific paradigms, it is placed at a crossroads of various other branches of knowledge. In the case of statistical mechanics, the standard theory - hereafter referred to as the Boltzmann-Gibbs (BG) statistical mechanics - exhibits highly relevant connections at a variety of microscopic, mesoscopic and macroscopic physical levels, as well as with the theory of probabilities (in particular, with the Central Limit Theorem, $C L T$ ). In many circumstances, the ubiquitous efects of the $C L T$, with its Gaussian attractors (in the space of the distributions of probabilities), are present. Within this complex ongoing frame, a possible generalization of the $B G$ theory was advanced in 1988 (C.T., J. Stat. Phys. 52, 479). The extension of the standard concepts is intended to be useful in those "pathological", and nevertheless very frequent, cases where the basic assumptions (molecular chaos hypothesis, ergodicity) for applicability of the $B G$ theory would be violated. Such appears to be, for instance, the case in classical long-range-interacting many-body Hamiltonian systems (at the so-called quasi-stationary state). Indeed, in such systems, the maximal Lyapunov exponent vanishes in the thermodynamic limit $N \rightarrow \infty$. This fact creates a quite novel situation with regard to typical $B G$ systems, which generically have a positive value for this central nonlinear dynamical quantity. This peculiarity has sensible effects at all physical micro-, meso- and macroscopic levels. It even poses deep challenges at the level of the $C L T$. In the present occasion, after 20 years of the 1988 proposal, we undertake here an overview of some selected successes of the approach, and of some interesting points that still remain as open questions. Various theoretical, experimental, observational and computational aspects will be addressed.
\end{abstract}

Keywords: Nonadditive entropy, Nonextensive statistical mechanics, Complex systems, Nonlinear dynamics

\section{INTRODUCTION}

Statistical mechanics indeed constitutes one of the pillars of contemporary physics. As such - together with mechanics (classical, quantum, relativistic), electromagnetism and thermodynamics - , it is one of the basic theories universally studied at intermediate and advanced levels in physics. As it happens with such grounding scientific paradigms, it plays a central role in various other branches of knowledge, including chemistry, biology, mathematics, computational sciences, to name but the most obvious ones. The standard theory hereafter referred to as the Boltzmann-Gibbs (BG) statistical mechanics - exhibits many connections at a variety of microscopic (mechanics, classical and quantum field theory, electrodynamics, nonlinear dynamical systems, gravitation), mesoscopic (Langevin, master equation, Fokker-Planck approaches, Vlasov equation) and macroscopic (thermodynamics, science of complexity) physical descriptions, as well as with mathematics (theory of probabilities, Central Limit Theorem, CLT).

Those physical approaches that are usually considered from first principles inescapable involve up to four independent and universal physical constants, namely the Planck constant $(h)$, the velocity of light in vacuum $(c)$, the gravitation constant $(G)$, and the Boltzmann constant $\left(k_{B}\right)$. All presently known physical units can be expressed as multiplicative expressions of powers of these four constants. Naturally, optimally formulated mathematics involve none of these constants, i.e., the exponents of all those powers vanish (which legitimates the common usage of units such that $h=c=G=$
$\left.k_{B}=1\right)$. The constant $k_{B}$ always appears, in one way or another, in statistical mechanics (e.g., in the laws of the ideal classical gas). In many instances, it is accompanied by the constant $h$ (e.g., in Fermi-Dirac and Bose-Einstein statistics). The constant $c$ can also be in the party (e.g., in the various forms expressing the laws of the black-body radiation). The constant $G$ accompanies $k_{B}$ whenever gravitation is taken into account (e.g., the variation of air density of the Earth atmosphere as a function of height, due to the gravitational mass attraction). Finally, all four constants $h, c, G$ and $k_{B}$ can be simultaneously present (e.g., in quantum gravitation thermostatistical expressions such as the entropy of a black-hole). In many of these and other circumstances, the efects of the CLT, with its Gaussian attractors (in the space of the distributions of probabilities), show up.

The most primitive form of gravitation (the $d=3$ Newtonian gravitation) emerges with $G \neq 0$. Maxwell equations and special relativity emerge with $1 / c \neq 0$. The most primitive form of quantum mechanics (the Schroedinger equation) emerges with $h \neq 0$. The basic form of statistical mechanics (based on the celebrated Boltzmann formula for the entropy, graved on his tomb in the Central Cemitery in Vienna) emerges with $1 / k_{B} \neq 0$ (and not with $k_{B} \neq 0$, as we shall see). The various possible combinations of these four universal constants (two by two, or three by three, or even all four together) can be represented as successively embedded tetrahedra. The $1 / k_{B}=0$ section of this structure is indicated in Fig. 1. The most general case (i.e., $\left(G, c^{-1}, h, k_{B}^{-1}\right) \neq(0,0,0,0)$ ) corresponds in principle to the statistical mechanics of quantum gravity, a theory yet to be constructed. 


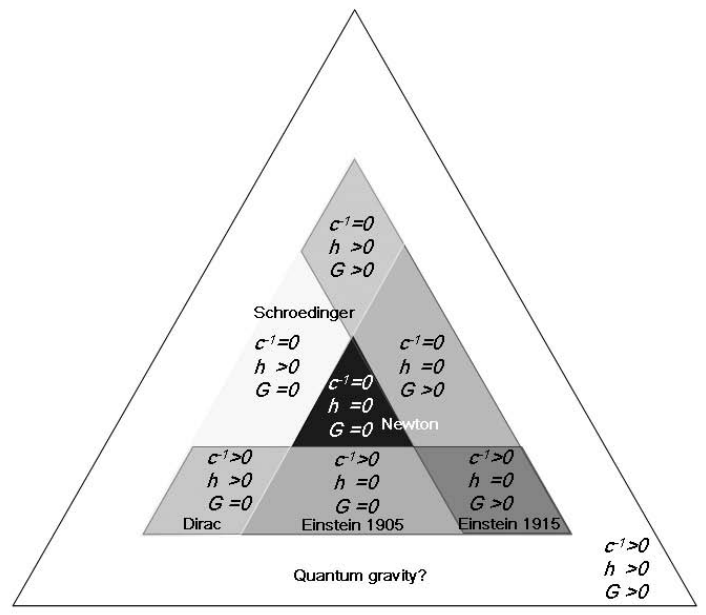

FIG. 1: Physical structure at the $k_{B}^{-1}=0$ plane. The full diagram involves four universal constants, and corresponds to successively embedded tetrahedra. At the center of the tetrahedron we have the case $G=c^{-1}=h=k_{B}^{-1}=0$, and the overall tetrahedron corresponds to $G>0, c^{-1}>0, h>0, k_{B}^{-1}>0$ (statistical mechanics of quantum gravity).

The above considerations are consistent with the fact that Planck introduced [1, 2] (see also [3-8]) four natural units for length, mass, time, and temperature, namely

$$
\begin{aligned}
& \text { unit of length } \quad=\sqrt{\frac{h G}{c^{3}}}=4.13 \times 10^{-33} \mathrm{~cm}(1) \\
& \text { unit of mass } \quad=\sqrt{\frac{h c}{G}}=5.56 \times 10^{-5} \mathrm{~g} \\
& \text { unit of time } \quad=\sqrt{\frac{h G}{c^{5}}}=1.38 \times 10^{-43} \mathrm{~s} \\
& \text { unit of temperature }=\frac{1}{k_{B}} \sqrt{\frac{h c^{5}}{G}}=3.50 \times 10^{32} \mathrm{~K}
\end{aligned}
$$

There is no need to add to this list the elementary electric charge $e$. Indeed, it is related to the already mentioned constants through the fine-structure constant $\alpha \equiv 2 \pi e^{2} / h c=$ $1 / 137.035999$...

In 1988, a generalization of the $B G$ theory was proposed [9], inspired by the structure of multifractals. It is based on the nonadditive entropy $S_{q}(q \in \mathcal{R})$, to be defined in the next Section, and is currently referred to as nonextensive statistical mechanics; it recovers the (additive) $B G$ entropy and its associated statistical mechanics as the $q=1$ particular instance. The extension of the standard concepts focuses on the frequent "pathological" cases where the basic assumptions (e.g., molecular chaos hypothesis, ergodicity) for applicability of the $B G$ theory are violated. One paradigmatic case concerns classical long-range-interacting many-body Hamiltonian systems (at the so-called quasi-stationary state). Indeed, in such systems, the maximal Lyapunov exponent vanishes in the thermodynamic limit $N \rightarrow \infty$. This fact is completely atypical within the $B G$ scenario. Indeed, most of the dynamical systems that have usually been studied, within the $B G$ theory, during the last 130 years generically have a positive value for the maximal Lyapunov exponent. This property has important consequences at physical micro-, meso- and macroscopic levels. Also, it sustains connections with the most important $C L T$. Last but not least, as we shall soon see, the quantity $(q-1)$ intriguingly couples to the universal constant $k_{B}$.

In Section 2, we briefly introduce the nonadditive entropy $S_{q}$, and its associated statistical mechanics. In Section 3, we show how the indice(s) $q$ are to be determined from microor meso-scopic information; we also describe typical mesoscopic mechanisms that are known to yield nonextensive statistics. In Section 4, we briefly illustrate the ubiquitous emergence of $q$-Gaussians (in general $q$-exponentials) in natural, artificial and social systems; consistently we present the $q$-generalization of the $C L T$, which can be thought as being the cause of this ubiquity. We finally conclude in Section 5 .

\section{NONADDITIVE ENTROPY AND NONEXTENSIVE STATISTICAL MECHANICS}

The entropic form introduced and studied by Boltzmann, Gibbs, von Neumann, Shannon and many others will from now on be referred to as the $B G$ entropy $S_{B G}$. For the discrete case, it is given by

$$
S_{B G}=-k \sum_{i=1}^{W} p_{i} \ln p_{i}
$$

with

$$
\sum_{i=1}^{W} p_{i}=1 \quad\left(p_{i} \in[0,1]\right)
$$

where $W$ is the number of possible microscopic states, and $k$ some conventional constant, typically taken to be $k_{B}$ in physics, and unity (or some other convenient dimensionless value) in computational sciences and elsewhere. For the particular case of equal probabilities (i.e., $p_{i}=1 / W, \forall i$ ), we immediately obtain the celebrated formula

$$
S_{B G}=k \ln W .
$$

The generalization of $S_{B G}$ proposed in [9] for generalizing $B G$ statistical mechanics is the following:

$$
S_{q}=k \frac{1-\sum_{i=1}^{W} p_{i}^{q}}{q-1} \quad\left(q \in \mathcal{R} ; S_{1}=S_{B G}\right) .
$$

For the particular case of equal probabilities, we obtain

$$
S_{q}=k \ln _{q} W,
$$

where the q-logarithmic function is defined as follows:

$$
\ln _{q} x \equiv \frac{x^{1-q}-1}{1-q} \quad\left(x>0 ; \ln _{1} x=\ln x\right) .
$$

Its inverse, the q-exponential function, is given by

$$
e_{q}^{x} \equiv[1+(1-q) x]_{+}^{\frac{1}{1-q}} \quad\left(e_{1}^{x}=e^{x}\right),
$$


where $[z]_{+} \equiv \max \{z, 0\}$.

The entropy (8) has various predecessors and related entropies. The (repeated) references to all these are largely diffused in the literature of nonextensive statistical mechanics. A summary can be found in [10].

It is worth mentioning that the entropy $S_{q}$ can be equivalently re-written as follows:

$$
S_{q}=k \sum_{i=1}^{W} p_{i} \ln _{q} \frac{1}{p_{i}}=-k \sum_{i=1}^{W} p_{i}^{q} \ln _{q} p_{i}=-k \sum_{i=1}^{W} p_{i} \ln _{2-q} p_{i}
$$

If we have two probabilistically independent systems $A$ and $B$, i.e. such that $p_{i j}^{(A+B)}=p_{i}^{(A)} p_{j}^{(B)}, \forall(i, j)$, we immediately verify

$$
\frac{S_{q}(A+B)}{k}=\frac{S_{q}(A)}{k}+\frac{S_{q}(B)}{k}+(1-q) \frac{S_{q}(A)}{k} \frac{S_{q}(B)}{k},
$$

hence

$$
S_{q}(A+B)=S_{q}(A)+S_{q}(B)+\frac{(1-q)}{k} S_{q}(A) S_{q}(B) .
$$

Therefore, for $q=1$, we recover the well known additivity of the $B G$ entropy, i.e.,

$$
S_{B G}(A+B)=S_{B G}(A)+S_{B G}(B),
$$

and this is so for any finite value of $k$. We also see that, for $q \neq 1, S_{q}$ is nonadditive. We further see that, for $q \neq 1$, additivity is asymptotically recovered in the limit $k \rightarrow \infty$. More precisely, it is asymptotically recovered for $(1-q) / k \rightarrow 0$, which creates a deep relationship between nonextensivity and the limit $k \rightarrow \infty$. Since the temperature $T$ is accompanied by $k$ (in the form $k T$ ) in any stationary-state distribution and any equation of states involving the temperature, this fact can be seen as the reason which makes the high-temperature asymptotic behavior of all known statistics (Boltzmann-Gibbs, Fermi-Dirac, Bose-Einstein, Gentile parastatistics, nonextensive statistics) to be one and the same, essentially the Maxwell-Boltzmann one.

The entropy $S_{q}$ can be, and has been, used in a great variety of situations for natural, artificial and social systems [11]. If the system is a physical one being described by a Hamiltonian, one can also develop a statistical mechanics (nonextensive statistical mechanics) as follows. Let us illustrate the procedure for the canonical ensemble (the system being in long-standing thermal contact with a large thermostat). We extremize $S_{q}$ with constraint (6), and also constraint [12]

$$
\langle\mathcal{H}\rangle_{q} \equiv \sum_{i=1}^{W} P_{i}^{(q)} E_{i}=U_{q}
$$

where the $\left\{E_{i}\right\}$ is the set of eigenvalues associated with the Hamiltonian $\mathcal{H}$ (and corresponding boundary conditions), $U_{q}$ is a fixed value characterizing the width of the distribution (we note that the width must always be a finite value, a property which is not guaranteed for the standard mean value, i.e. with probabilities $\left\{p_{i}\right\}$, if $q$ happens to be not close enough to unity), and

$$
P_{i}^{(q)} \equiv \frac{p_{i}^{q}}{\sum_{j=1}^{W} p_{j}^{q}}
$$

is referred to as the escort distribution. It follows straightforwardly that

$$
p_{i} \equiv \frac{\left[P_{i}^{(q)}\right]^{\frac{1}{q}}}{\sum_{j=1}^{W}\left[P_{j}^{(q)}\right]^{\frac{1}{q}}} .
$$

This optimization procedure yields, for the stationary state,

$$
p_{i}=\frac{e_{q}^{-\beta_{q}\left(E_{i}-U_{q}\right)}}{\sum_{j=1}^{W} e_{q}^{-\beta_{q}\left(E_{j}-U_{q}\right)}},
$$

with

$$
\beta_{q}=\frac{\beta}{\sum_{j=1}^{W} p_{j}^{q}},
$$

$\beta$ being the Lagrange parameter associated with constraint (16). Further details, as well as the connections with thermodynamics, can be seen in $[12,13]$.

The form of the energy constraint (16), using $P_{i}^{(q)}$ instead of the usual $p_{i}$ can be understood along various convergent lines. Let us restrict here to mentioning that both the norm and the energy constraints are well defined (i.e., corresponding to finite values) for $q$ below some critical value, and both diverge for $q$ equal or above that value. In the absence of degeneracy, this critical value is $q=2$ (note that the standard mean value of the energy is finite only up to $q=3 / 2$, being infinite for $q \geq 3 / 2$ ). The use of the $q$-expectation values such as (16) has been recently criticized in [14]. This critique has been replied in $[15,16]$. Further arguments that could suggest the use of $q$-expectation values can be found in [10], and also in [17-24]. Other arguments that could suggest the use of standard averages can be found in [24-26]. This particular issue is somewhat unclear at the present date. Indeed, in addition to the various apparently contradicting arguments, calculations also exist which suggest the equivalence of both procedures (either using, for the energy constraint, averages with $\left\{p_{i}\right\}$, or using averages with $\left\{P_{i}\right\}$ whenever the former ones are finite) $[12,27]$. To illustrate however a basic point, let us assume that the variable $x$ runs from zero to infinity, and that the energy is proportional to $x^{\sigma}(\sigma>0)$. The stationary-state exhibits therefore a probability $p(x) \propto e_{q}^{-\beta x^{\sigma}}$, hence, asymptotically for $x \rightarrow \infty, p(x) \sim x^{-\sigma /(q-1)}$. This distribution (i) is normalizable only for $q<1+\sigma$ (hence $q<2$ for $\sigma=1$, and $q<3$ for $\sigma=2$ ); (ii) satisfies that $\left\langle x^{\sigma}\right\rangle$ is finite only for $q<(1+2 \sigma) /(1+\sigma)$ (hence $q<3 / 2$ for $\sigma=1$, and $q<5 / 3$ for $\sigma=2$ ); (iii) satisfies that $\left\langle x^{\sigma}\right\rangle_{q}$ is finite only for $q<1+\sigma$, which coincides with the upper bound for normalizability. In other words, if we use $q$-expectation values, the entire theory is valid up to $q=1+\sigma$, whereas if we use standard expectation values, the norm-constraint is mathematically admissible up to $q=1+\sigma$, and the energy-constraint is mathematically admissible up to a lower value, namely $(1+2 \sigma) /(1+\sigma)$.

Many applications of nonextensive statistical mechanics can be found in [28-41] and elsewhere [11]. Along the years, many areas have been focused on such as theory of finance [42], anomalous diffusion [43], magnetism [44], stochastic equations [45], biological evolution [46], turbulence in pureelectron plasma [47], among others. 


\section{DETERMINING THE INDICES $q$, AND THE EXTENSIVITY OF THE NONADDITIVE ENTROPY $S_{q}$ FOR COMPLEX SYSTEMS}

\subsection{Sets of indices $q$}

The theory advanced in the previous Section is in principle valid for arbitray values of the index $q$. We shall now address the following essential question: For a given specific system or class of systems, how can we determine the values of its indice (s) $q$ ? To start, let us point out that, for a given system, not one but a whole family of (possibly infinite countable) indices $\{q\}$ is to be determined. Let us give a few examples:

$q_{\text {sensitivity }}$ : A dissipative one-dimensional (or, say, conservative two-dimensional) nonlinear dynamical system $x(t)$ typically exhibits a sensitivity to the initial conditions $\xi \equiv$ $\lim _{\Delta x(0) \rightarrow 0} \frac{\Delta x(t)}{\Delta x(0)}$ of the form

$$
\xi=e_{q_{\text {sensitivity }}}^{\lambda_{q_{\text {sensitity }}}{ }^{2}},
$$

where $\lambda_{q_{\text {sensitivity }}}$ is the $q$-generalized Lyapunov coefficient. A practical numerical manner for determining it consists in plotting $\ln _{q} \xi(t)$ versus $t$ for various values of $q$ until a value is found so that, asymptotically in the $t \rightarrow \infty$ limit, $\ln _{q} \xi(t) \propto t$. That value of $q$ is $q_{\text {sensitivity }}$, and the slope is $\lambda_{q_{\text {sensitivity }}}$. The two most interesting situations occur for strong chaos (i.e., when the Lyapunov exponent $\lambda_{1}$ is positive), and for weak chaos (at the edge of chaos, where $\lambda_{1}=0$ and $\lambda_{q_{\text {sensitivity }}}>0$ ). In the former case we have $q_{\text {sensitivity }}=1$; in the latter $q_{\text {sensitivity }}<1$. Although not particularly relevant within the present context, $q_{\text {sensitivity }}>1$ can also occur. Such is the case at double-period and tangent bifurcation critical points.

$q_{\text {entropy production }}$ : The phase space of a $d$-dimensional nonlinear dynamical system is partitioned into many $W$ cells. We put, in one of those cells (chosen randomly, or in any other convenient manner), $M$ initial conditions, and then let these points spread around. We define $p_{i}(t) \equiv M_{i}(t) / M$ $(i=1,2,3, \ldots, W)$, where $M_{i}(t)$ is the number of points within the $i$-th cell at time $t\left(\sum_{i=1}^{W} M_{i}(t)=M, \forall t\right)$. We then calculate, with these $\left\{p_{i}(t)\right\}$ into Eq. (8), $S_{q}(t) / k$. We will verify that, for a huge class of nonlinear dynamical systems, a value of $q$ exists such that $S_{q}(t) \propto t$. That value of $q$ is $q_{\text {entropy production }}$, and the slope is the $q$-generalized Kolmogorov-Sinai-like entropy production rate $K_{q_{\text {entropy production }}}$. To be more precise, we have

$$
\lim _{t \rightarrow \infty} \lim _{W \rightarrow \infty} \lim _{M \rightarrow \infty} \frac{S_{q_{\text {entropy production }}}(t)}{k t}=K_{q_{\text {entropy production }}}
$$

In the presence of strong chaos (weak chaos) we have $q_{\text {entropy production }}=1$ ( $\left.q_{\text {entropy production }}<1\right)$. For $d=1$ systems, we expect $q_{\text {entropy production }}=q_{\text {sensitivity }}$ and $K_{q_{\text {entropy production }}}=\lambda_{q_{\text {sensitivity }}}$, thus verifying a Pesin-like identity.

$q_{\text {relaxation }}$ : Various procedures have been used to determine this index. Let us describe one particularly simple case, namely that of a $d$-dimensional dissipative nonlinear dynamical system. We partition its phase space in $W$ cells, and use a large number of initial conditions uniformly spread in all these cells. Then count the number of cells $W(t)$ within which there exists at least one point at time $t$. The system being dissipative, and for $W$ increasingly large, one expects

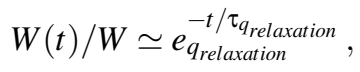

which defines the index $q_{\text {relaxation. }}$. For strong chaos (weak chaos) we expect $q_{\text {relaxation }}=1\left(q_{\text {relaxation }}>1\right)$. The computational procedure that we have just described is not the possibly most precise one, but is has the advantage of being easily implemented.

$q_{\text {stationarystate }}$ : This index is the one characterizing the distribution of energies for the stationary state (which coincides with thermal equilibrium for $q=1$ ). In other words,

$$
p_{i} \propto e_{q_{\text {stationary state }}}^{-\beta_{q_{\text {stationary state }}} E_{i}} .
$$

Although no proof is available, this index might coincide (at least for a large class of systems) with that of the distribution of velocities $v_{i}$, i.e., $p\left(v_{i}\right) \propto e_{q_{\text {stationary state }}^{-B v_{i}^{2}} \text {. It might }}$ also coincide with the index noted $q_{\text {limit }}$ in the literature, where limit refers in the sense of $N \rightarrow \infty$, where $N$ is the number of particles of a probabilistic system; it might also coincide with $q_{\text {attractor }}$, where attractor is used in the sense of the central limit theorem. It is not even excluded that it coincides with the index characterizing some correlation functions (e.g., velocity-velocity autocorrelation function or others). At thermal equilibrium we have $q_{\text {stationary state }}=1$; for more complex stationary states, one typically expects $q_{\text {stationary state }}>1$ (although the possibility $q_{\text {stationary state }}<1$ is by no means excluded).

$q_{\text {entropy }}:$ In order to be consistent with clasical thermodynamics, the entropy of a system composed of $N$ elements should be extensive, i.e., asymptotically proportional to $N$ in the $N \rightarrow \infty$ limit. There is a plethora of systems for which a value of $q$ exists which satisfies this requirement. That value of $q$ is $q_{\text {entropy }}$, i.e.,

$$
0<\lim _{N \rightarrow \infty} \frac{S_{q_{\text {entropy }}}(N)}{N}<\infty .
$$

If there are no correlations or if they are weak, we have $q_{\text {entropy }}=1$; if the correlations are strong we typically (but not necessarily) have $q_{\text {entropy }}<1$. We remind however that "pathological" systems exist for which no value of $q$ succeeds in making the entropy extensive.

Let us illustrate these features for the simple case of equal probabilities, i.e., $p_{i}=1 / W, \forall i$. If, in the limit $N \rightarrow \infty, W(N) \sim A \mu^{N}(\mu>1 ; A>0)$, we have $q_{\text {entropy }}=1$. If we have $W(N) \sim B N^{\rho}(\rho>0 ; B>0)$, then $q_{\text {entropy }}=1-\frac{1}{\rho}<1$. As a final example, let us assume that $W(N) \sim C \mu^{N \gamma}(\mu>1 ; 0<\gamma<1 ; C>0)$. In such a case, no value of $q$ exists which could produce $S_{q}(N) \propto N$.

$q_{\text {correlation }}$ : This index characterizes a strong correlation involved in the system. It is sometimes introduced through the q-product $[48,49]$

$$
x \otimes_{q} y \equiv\left[x^{1-q}+y^{1-q}-1\right]_{+}^{\frac{1}{1-q}} \quad(x \geq 0, y \geq 0),
$$


which satisfies $x \otimes_{1} y=x y$, and the extensive property $\ln _{q}\left(x \otimes_{q} y\right)=\ln _{q} x+\ln _{q} y$ [to be compared with the nonadditive property $\left.\ln _{q}(x y)=\ln _{q} x+\ln _{q} y+(1-q)\left(\ln _{q} x\right)\left(\ln _{q} y\right)\right]$.

Strong correlations can be also introduced through nonlinearity and/or inhomogeneity in differential equations. Absence of correlation (i.e., probabilistic independence) corresponds to $q_{\text {correlation }}=1$. Presence of strong correlations corresponds to $q_{\text {correlation }} \neq 1$.

\subsection{Examples of indices $q$}

We may consider nonextensivity universality classes of systems, e.g., Hamiltonian many-body systems having twobody interactions decaying (attractively) with distance as $1 /$ distance $^{\alpha}$. All systems sharing the same $\alpha$ (and perhaps some other common features) possibly have the same set of $q$ 's. The same occurs for say families of one-dimensional unimodal dissipative maps sharing the same inflexion at the extremum.

The various indices can be determined (in principle analytically) from microscopic information about the system, typically its nonlinear dynamics (e.g., deterministic maps, Hamiltonian systems) or its complete probabilistic description (e.g., the full set of probabilities of the possible configurations of $N$ discrete or continuous random variables, either independent or correlated), or from mesoscopic information. In the latter case, at least two important mechanisms leading to nonextensive statistics have been identified, namely a nonlinear homogeneous Fokker-Planck equation [50, 51], associated with a strongly non-Markovian Langevin equation [52], and a linear inhomogeneous Fokker-Planck equation [45, 53], associated with multiplicative noise Langevin equation [45, 53]. Both mechanisms can be unified within a more general (simultaneously nonlinear and inhomogeous) Fokker-Planck equation [54], essentially implying a long-range memory. The various connections of statistical mechanics (either $B G$ or nonextensive) and other important approaches are schematically described in Fig. 2.

Let us illustrate the various facts mentioned above through some selected examples.

(i) For the dissipative one-dimensional unimodal maps belonging to the $z$-logistic or the $z$-circular classes we have $q_{\text {sensitivity }}(z)=q_{\text {entropy production }}(z)$ satisfying

$$
\frac{1}{1-q_{\text {sensitivity }}(z)}=\frac{1}{\alpha_{\min }(z)}-\frac{1}{\alpha_{\max }(z)} \quad\left(q_{\text {sensitivity }}(z)<1\right)
$$

where $\alpha_{\min }(z)$ and $\alpha_{\max }(z)$ are respectively the minimal and maximal values of $\alpha$ for which the multifractal function $f(\alpha)$ vanishes. It is known that

$$
\begin{aligned}
\alpha_{\text {max }}(z) & =\frac{\ln b}{\ln \alpha_{F}(z)} \\
\alpha_{\text {min }}(z) & =\frac{\ln b}{z \ln \alpha_{F}(z)}
\end{aligned}
$$

where $\alpha_{F}(z)$ is the $z$-generalized Feigenbaum constant of the specific universality class of maps, and $b$ is the partition scale
( $b=2$ for the $\mathrm{z}$-logistic maps; $b=(\sqrt{5}+1) / 2=1.6180 \ldots$, the golden mean, for the z-circular maps). Hence

$$
\frac{1}{1-q_{\text {sensitivity }}(z)}=(z-1) \frac{\ln \alpha_{F}(z)}{\ln b} .
$$

Broadhurst calculated the $z=2$ logistic map Feigenbaum constant $\alpha_{F}(2)$ with 1,018 digits [56]. Hence, it straighforwardly follows that

$$
q_{\text {sensitivity }}(2)=0.244487701341282066198 \ldots \ldots
$$

(ii) For the block entropy corresponding to an infinitelylong linear chain with ferromagnetic first-neighbor interactions belonging to the universality class characterized by the central charge $c$, at the $T=0$ quantum critical point in the presence of a transverse magnetic field, it has been established [57] that

$$
q_{\text {entropy }}(c)=\frac{\sqrt{9+c^{2}}-3}{c} .
$$

Therefore, for the Ising model, we have $q_{\text {entropy }}(1 / 2)=$ $\sqrt{37}-6=0.08 \ldots$, for the isotropic $X Y$ model, we have $q_{\text {entropy }}(1)=\sqrt{10}-3=0.16 \ldots$, and for the $c \rightarrow \infty$ limit we have $q_{\text {entropy }}(\infty)=1$, i.e., the $B G$ result. The physical interpretation of this interesting limit remains elusive. See Fig. 3.

iii) A probabilistic model with $N$ binary equal random variables can be represented as a triangle having, for fixed $N,(N+1)$ different elements with multiplicities respectively given by $\frac{N !}{N ! 0 !}=1, \frac{N !}{(N-1) ! 1 !}=N, \frac{N !}{(N-2) ! 2 !}=\frac{N(N-1)}{2}, \ldots, \frac{N !}{0 ! N !}=$ 1. Strong correlations can be introduced [58] so that, for given $N$, only the first $(d+1)$ elements (of the $(N+1)$ possible ones) have nonvanishing probabilities, all the other $(N-d)$ elements of the same row having zero probability to occur. It has been shown for this specific model, which turns out to be asymptotically scale-invariant, that

$$
q_{\text {entropy }}=1-\frac{1}{d} \quad(d=1,2,3, \ldots) .
$$

(iv) A probabilistic model with $N$ correlated binary variables which strictly satisfies scale invariance (i.e., the triangle Leibnitz rule) has been introduced in [59], whose $N \rightarrow \infty$ limiting distribution (appropriately centered and scaled) is a $q_{\text {limit }}$-Gaussian with

$$
q_{\text {limit }}=\frac{\mathrm{v}-2}{\mathrm{v}-1} \quad(\mathrm{v}=1,2,3, \ldots) .
$$

The index $v$ characterizes a particular triangle (with particular strong correlations); $v=1$ corresponds to the Leibnitz triangle itself; $v \rightarrow \infty$ corresponds to independent random variables. This model has been further generalized [60], in such a way that $q_{\text {limit }}$ is still given by (33) but $v$ being now a real (not necessarily integer) number $v \geq 1$ (hence $q_{\text {limit }} \leq 1$ ). Along the same lines, the model can be modified in such a way that the region $q_{\text {limit }}>1$ becomes admissible as well, while still 


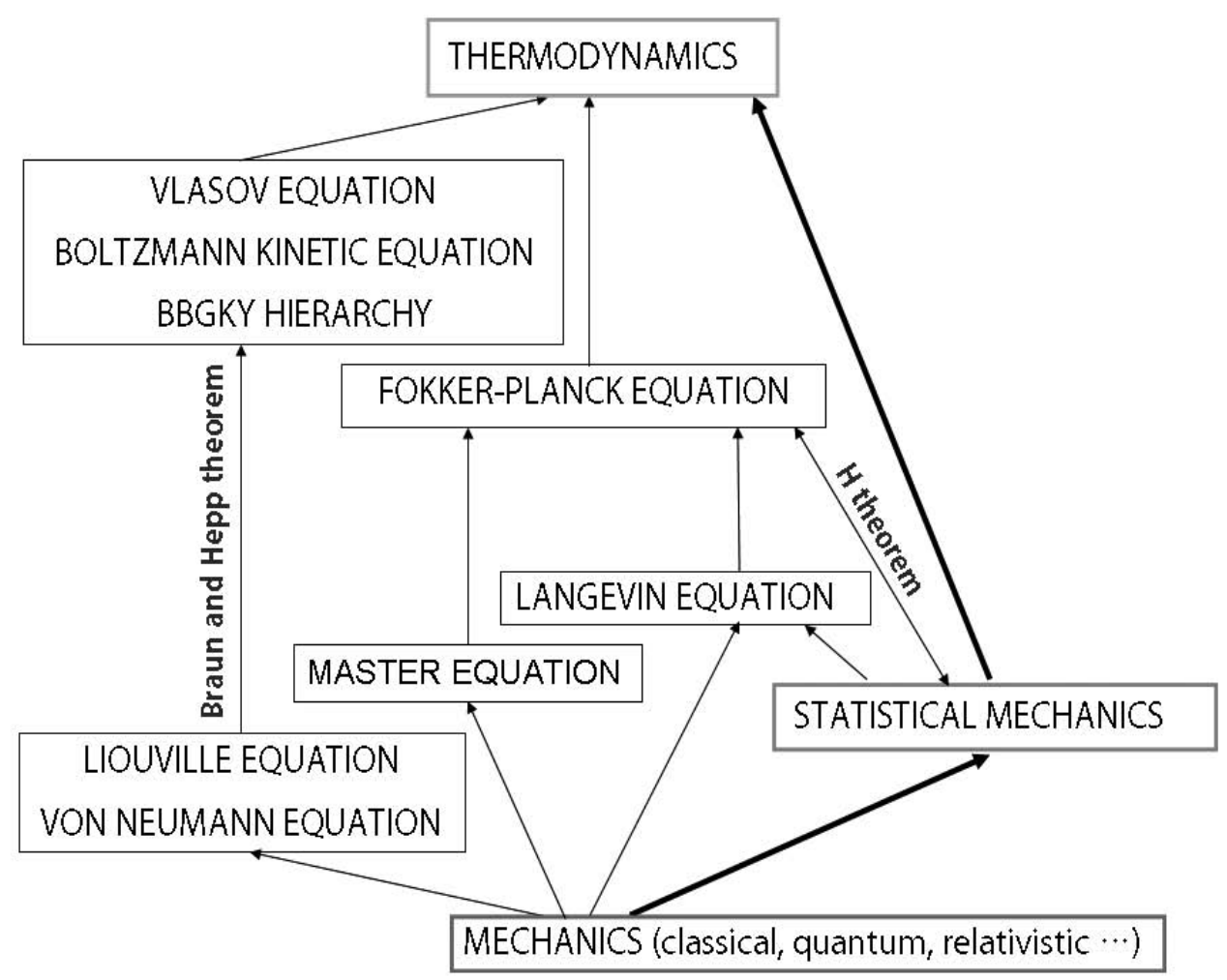

FIG. 2: Main paths of thermal physics: from mechanics (microscopic level) to thermodynamics (macroscopic level), through Langevin, Fokker-Planck, master, Liouville, von Neumann, Vlasov, Boltzmann kinetic, BBGKY hierarchy equations (mesoscopic level). The crucial connection with statistical mechanics (Boltzmann-Gibbs theory, nonextensive statistical mechanics, or others) is done through the knowledge of the values of indices such as the $q$ 's (typically a family of infinite countable interconnected values of $q$ ). If we have full knowledge at the dynamical (mechanical) level, i.e., the time evolution of the system in say phase space, the relevant $q$ 's $\left(q_{\text {sensitivity }}, q_{\text {entropy production }}, q_{\text {relaxation }}\right.$, $q_{\text {stationary state }}, q_{\text {entropy }}$, etc) can be calculated from first principles; such is the case of unimodal dissipative maps like the logistic one, classical two-body-interacting many-body Hamiltonian systems, strongly quantum-entangled Hamiltonian systems, etc, although the calculation might sometimes (quite often in fact!) be mathematically or numerically untractable. If we only have information at the mesoscopic level (e.g., types and amplitudes of involved noises, linear or nonlinear coefficients and exponents, etc), the relevant $q$ 's can in principle be analytically determined as functions of those coefficients and exponents; such is the case of the multiplicative- and additive-noise Langevin equation, the linear heterogenous and the nonlinear homogeneous Fokker-Planck equations, and similar ones. If we lack full information at both microscopic and mesoscopic levels, we may still obtain useful information by proceeding through careful fittings of experimental and/or observational and/or numerical data, by using appropriate functional forms ( $q$-exponential, $q$-Gaussians, or even more general or different forms). For the Braun and Hepp theorem connection, see [55]; for the $H$ theorem connection, see [24] and references therein.

being strictly scale invariant. For this family of models, it can be also proved that $q_{\text {entropy }}=1$ (see in Fig. 4 an illustration corresponding to $q_{\text {limit }}=1.5$ ).

(v) A phenomenological model based on a linear inhomogeneous Fokker-Planck equation has been suggested by Lutz [61] to describe the velocity distribution of cold atoms in dissipative optical lattices. He obtained that the distribution should be a $q_{\text {stationart state }}$-Gaussian with

$$
q_{\text {stationary state }}=1+\frac{44 E_{R}}{U_{0}} \geq 1,
$$

where $E_{R}$ is the recoil energy, and $U_{0}$ is the potential depth. Later on, we will come back onto this prediction.

(vi) The solution corresponding to the Langevin equation including multiplicative noise mentioned previously [45] is a $q_{\text {stationary state }}-$ Gaussian with

$$
q_{\text {stationary state }}=\frac{\tau+3 M}{\tau+M} \geq 1,
$$

where $\tau$ and $M$ are parameters of the phenomenological model. In particular, $M$ is the amplitude of the multiplicative noise. We verify that $M=0$ yields $q_{\text {stationary state }}=1$, whereas $M>0$ yields $q_{\text {stationary state }}>1$.

(vii) A phenomenological model based on a Langevin equation including colored symmetric dichotomous noise [62] has as solution a $q_{\text {stationary state }}$-Gaussian with

$$
q_{\text {stationary state }}=\frac{1-2 \gamma / \lambda}{1-\gamma / \lambda},
$$

where $(\gamma, \lambda)$ are parameters of the model. We verify that $\gamma / \lambda=0$ yields $q_{\text {stationary state }}=1$, whereas $\gamma / \lambda>0$ yields 


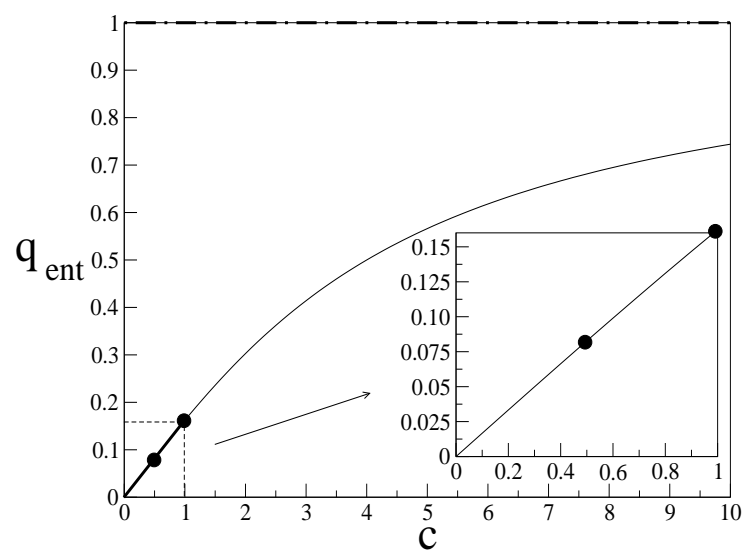

FIG. 3: $q_{\text {entropy }}$ as a function of the central charge $c$. It asymptotically approaches the $B G$ limit $q_{\text {entropy }}=1$ when $c \rightarrow \infty$. From [57].

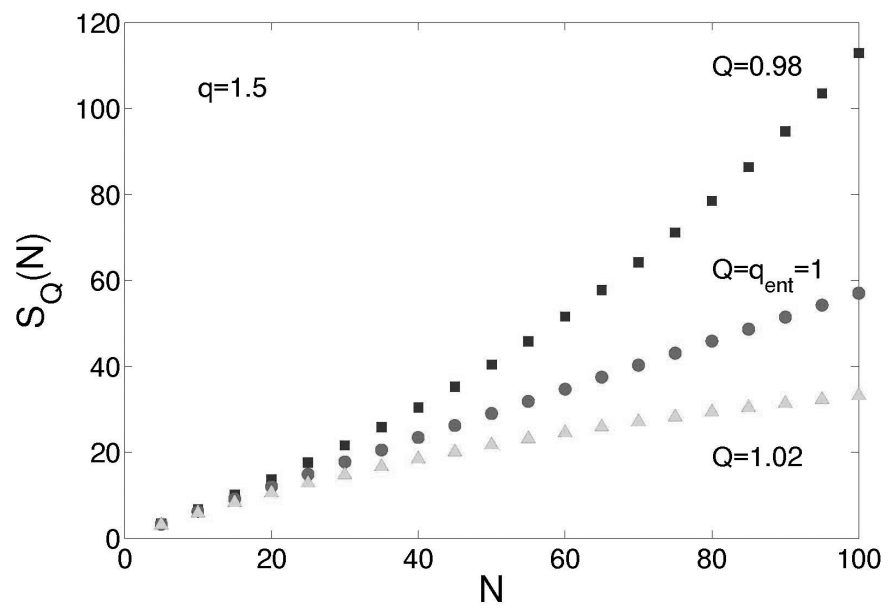

FIG. 4: $S_{Q}$ for various values of $Q$ for the probabilistic model with $N$ correlated binary variables in [60], corresponding to $q_{\text {limit }}=1.5$. We numerically verify the analytical result $q_{\text {entropy }}=1$, i.e., that, for this model, only $S_{B G}(N)$ is extensive.

$q_{\text {stationary state }}<1$.

(viii) A Ginzburg-Landau discussion of point kinetics for $n=d$ ferromagnets yields $[63,64]$ a $q_{\text {stationary state }}$-Gaussian distribution of velocities with

$$
q_{\text {stationary state }}=\frac{d+4}{d+2} \geq 1 \text {. }
$$

We verify that when $d$ increases from zero to $\infty$, $q_{\text {stationary state }}=1$ decreases from 2 to unity.

(ix) A growth model for a stochastic network (belonging to the so called scale-free class) based on preferential attachment has been proposed in [65]. The degree distribution is given by the $q_{\text {stationary state }}$-exponential form $p(k)=$ $p(0) e_{q_{\text {stationary state }}^{-k / \kappa}(\kappa>0)}(\kappa)$ with (see [10] for details about the transformation from the form given in [65] to the $q$ -

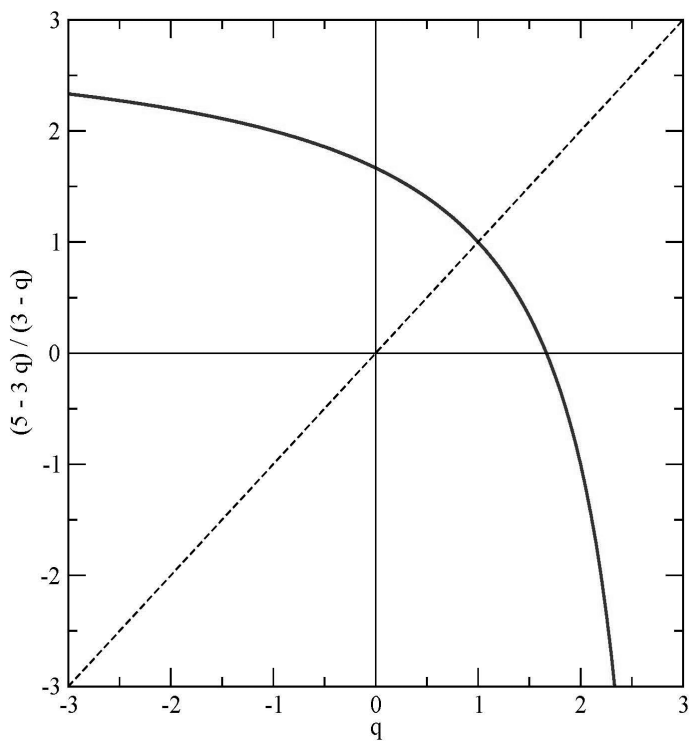

FIG. 5: $q_{1}^{-}$as a function of $q \equiv q_{0}$, as given by Eq. (58) (from [76]).

exponential form)

$$
q_{\text {stationary state }}=\frac{2 m(2-r)+1-p-r}{m(3-2 r)+1-p-r} \geq 1,
$$

where $(m, p, r)$ are parameters of the model.

The above examples paradigmatically illustrate how $q$ can be determined from either microscopic or mesoscopic information. Further analytical expressions for $q$ in a variety of other physical systems are presented in [66-70].

\subsection{Connections between indices $q$}

The full understanding of all the possible connections between these different $q$-indices still remains elusive. Many examples exist for which one or more of these $q$ 's are (analytically or numerically) known and understood. However, the complexity of this question has not yet allowed for transparent, complete and general understanding.

Nevertheless, at the light of what is presently known, the scenario appears to be that, for a given system, a countable set of $q$ 's can exist, each of them being basically associated with a specific (more or less important) property of the system. Let us denote this set with $\left\{q_{m}\right\}(m=0, \pm 1, \pm 2, \ldots)$. For many systems, if not all, the structure seems to be such that very few (typically only one) of those $q$ 's are independent, all the others being functions of those few. Let us illustrate what we mean by assuming that only one is independent, and let us denote it by $q_{0}$. So, we typically have

$$
q_{m}=f_{m}\left(q_{0}\right) \quad\left(m=0, \pm 1, \pm 2, \ldots ; f_{0}\left(q_{0}\right)=q_{0}\right) .
$$

The form of $f_{m}\left(x_{0}\right)$ (or of similar functions) can be quite complex (see, for instance, $[71,72]$ ). However, intriguingly enough, very many among them seem to conform to the fol- 

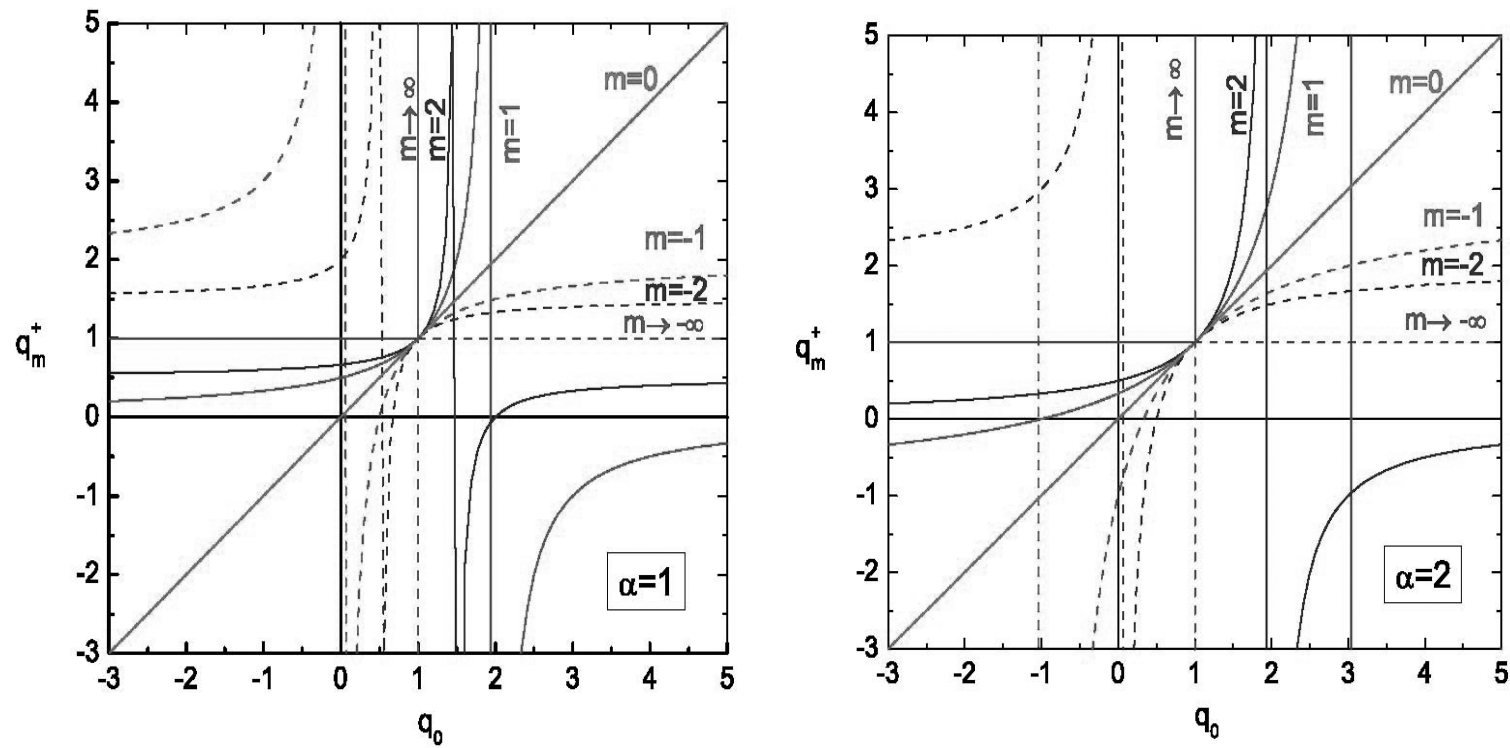

FIG. 6: $q_{m}^{+}$as a function of $q_{0}^{+} \equiv q_{0}$ for typical values of $m$. For clarity, the vertical asymptotes are indicated as well. Left: $\alpha=1 ;$ Right: $\alpha=2$. For each value $m>0$, there are two branches: the left one is such that $q_{m}^{+}>1\left(q_{m}^{+}<1\right)$ if $q_{0}>1\left(q_{0}<1\right)$; the right one does not contain the point $\left(q_{0}, q_{m}^{+}\right)=(1,1)$. For each value $m<0$, there are two branches: the right one is such that $q_{m}^{+}>1\left(q_{m}^{+}<1\right)$ if $q_{0}>1\left(q_{0}<1\right)$; the left one does not contain the point $\left(q_{0}, q_{m}^{+}\right)=(1,1)$.
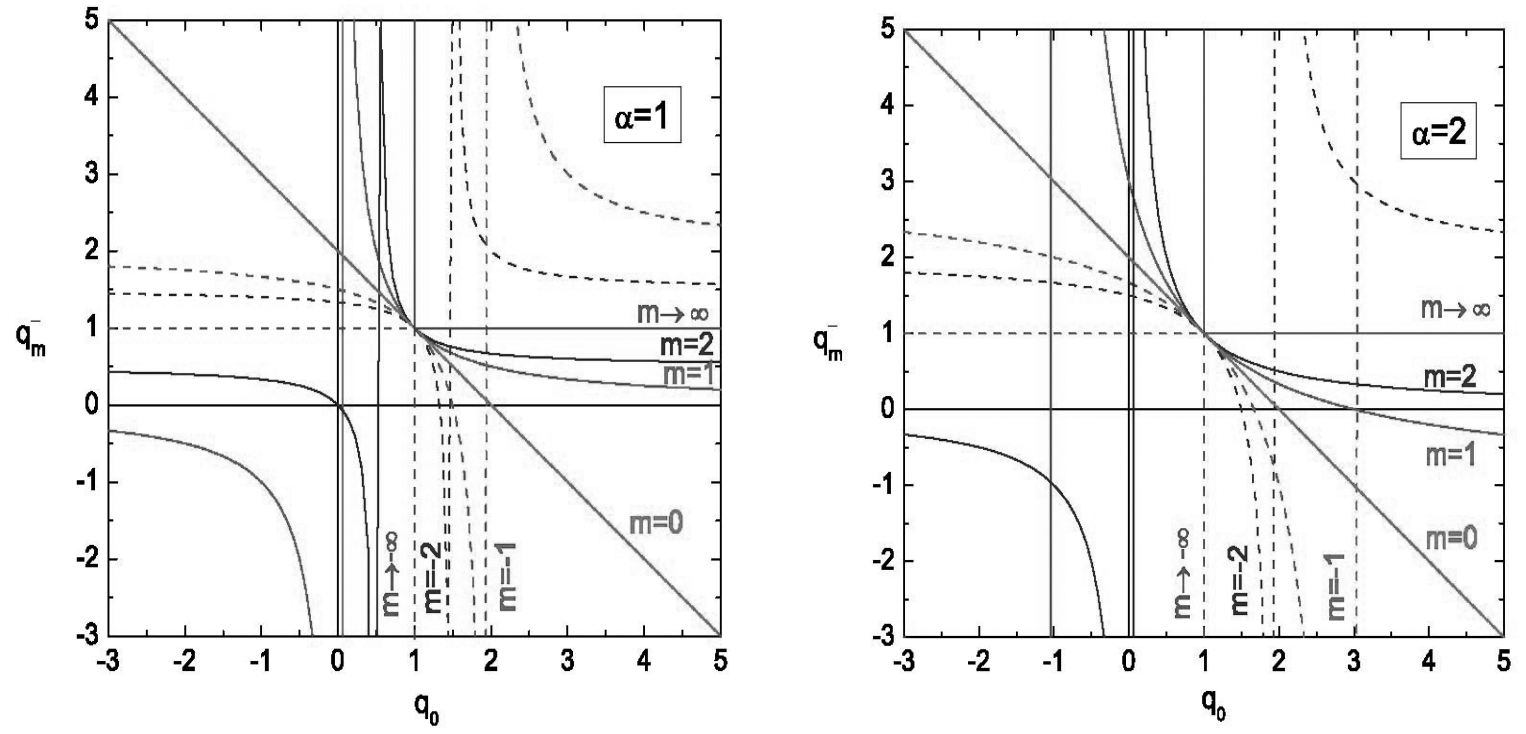

FIG. 7: $q_{m}^{-}$as a function of $q_{0}^{+} \equiv q_{0}$ for typical values of $m$. For clarity, the vertical asymptotes are indicated as well. Left: $\alpha=1 ;$ Right: $\alpha=2$. For each value $m>0$, there are two branches: the right one is such that $q_{m}^{+}>1\left(q_{m}^{+}<1\right)$ if $q_{0}<1\left(q_{0}>1\right)$; the left one does not contain the point $\left(q_{0}, q_{m}^{+}\right)=(1,1)$. For each value $m<0$, there are two branches: the left one is such that $q_{m}^{+}>1\left(q_{m}^{+}<1\right)$ if $q_{0}<1\left(q_{0}>1\right)$; the right one does not contain the point $\left(q_{0}, q_{m}^{+}\right)=(1,1)$.

lowing simple structure. In some case $q_{m}$ satisfies

$$
\frac{\alpha}{1-q_{m}^{+}}=\frac{\alpha}{1-q_{0}}+m \quad(m=0, \pm 1, \pm 2, \ldots),
$$

where $0<\alpha \leq 2$. In other cases it satisfies, through the so- called additive duality $q_{0} \leftrightarrow\left(2-q_{0}\right)$,

$$
\frac{\alpha}{1-q_{m}^{-}}=\frac{\alpha}{q_{0}-1}+m \quad(m=0, \pm 1, \pm 2, \ldots),
$$

where again $0<\alpha \leq 2$. We easily see that, if we apply again this transformation, we go back to the initial value $q_{0}$. In other 
words, for fixed $(\alpha, m)$, we have that

$$
q_{m}^{-}\left(q_{m}^{-}\left(q_{0}\right)\right)=q_{0}, \forall q_{0} .
$$

Or equivalently, the function $q_{m}^{-}\left(q_{0}\right)$ concides with the function $q_{0}\left(q_{m}^{-}\right)$.

It respectively follows from (40) and (41) that

$$
\frac{\alpha}{1-q_{m}^{+}}-m=\frac{\alpha}{1-q_{m^{\prime}}^{+}}-m^{\prime} \quad\left(m, m^{\prime}=0, \pm 1, \pm 2, \ldots\right)
$$

and

$$
\frac{\alpha}{1-q_{m}^{-}}-m=\frac{\alpha}{1-q_{m^{\prime}}^{-}}-m^{\prime} \quad\left(m, m^{\prime}=0, \pm 1, \pm 2, \ldots\right) \text {. }
$$

If we define $q_{m}^{+}\left(q_{0}\right)$ through Eq. (40) and $q_{m}^{-}\left(q_{0}\right)$ through Eq. (41), we easily see that

$$
q_{m}^{-}\left(q_{0}\right)=q_{m}^{+}\left(2-q_{0}\right) .
$$

If we make an analytical extension in (40) such that $m$ and $m^{\prime}$ are allowed to be real numbers, we may consider the case

$$
m^{\prime}=m-\alpha \quad(0<\alpha \leq 2)
$$

By replacing this into Eqs. (43) and (44), we respectively obtain

$$
q_{m-\alpha}^{+}=2-\frac{1}{q_{m}^{+}}
$$

and

$$
q_{m-\alpha}^{-}=2-\frac{1}{q_{m}^{-}} .
$$

This specific form of connection appears in very many occasions in nonextensive statistical mechanics. In particular, if $\alpha=1$ we have

$$
q_{m-1}^{+}=2-\frac{1}{q_{m}^{+}}
$$

and

$$
q_{m-1}^{-}=2-\frac{1}{q_{m}^{-}} .
$$

And if $\alpha=2$ we have

$$
q_{m-2}^{+}=2-\frac{1}{q_{m}^{+}} .
$$

and

$$
q_{m-2}^{-}=2-\frac{1}{q_{m}^{-}} .
$$

The families (40) and (41) can be respectively rewritten as follows:

$$
q_{m}^{+}=\frac{\alpha q_{0}+\left(1-q_{0}\right) m}{\alpha+\left(1-q_{0}\right) m} \quad(m=0, \pm 1, \pm 2, \ldots),
$$

and

$$
q_{m}^{-}=\frac{\alpha\left(2-q_{0}\right)+\left(q_{0}-1\right) m}{\alpha+\left(q_{0}-1\right) m} \quad(m=0, \pm 1, \pm 2, \ldots) .
$$

In both cases we verify that $q_{0}=1$ yields $q_{m}=1, \forall m$, and that $q_{ \pm \infty}^{ \pm}=1, \forall q_{0} \neq 1$. For one of the two branches of the family (53) we verify that $q_{0}>1$ implies $q_{m}^{+}>1, \forall m$, and that $q_{0}<1$ implies $q_{m}^{+}<1, \forall m$. For one of the two branches of the family (54) it is the other way around, i.e, $q_{0}>1$ implies $q_{m}^{-}<1, \forall m$, and $q_{0}<1$ implies $q_{m}^{-}>1, \forall m$. It is possible to simultaneously write both families in a compact manner, namely

$$
q_{m}^{ \pm}=\frac{\alpha\left[1 \pm\left(q_{0}-1\right)\right] \pm\left(1-q_{0}\right) m}{\alpha \pm\left(1-q_{0}\right) m} \quad(m=0, \pm 1, \pm 2, \ldots) .
$$

These relations exhibit a vertical asymptote at $q_{0}=1 \pm \frac{\alpha}{m}$, and a horizontal asymptote at $q_{m}^{ \pm}=1 \mp \frac{\alpha}{m}$.

It is also worth stressing two interesting properties of $q_{m}^{-}$.

First, we verify that

$$
q_{0}^{-}=2-q_{0},
$$

which precisely recovers the already mentioned additive $d u$ ality. This duality admits only one fixed point, namely $q_{0}=1$.

Second, we verify that

$$
\lim _{m \rightarrow \alpha} q_{m}^{-}=\frac{1}{q_{0}}
$$

which precisely is the so-called multiplicative duality. This duality admits two fixed points, namely $q_{0}=1$ and $q_{0}=-1$.

It can be shown $[10,58]$ that, by successively and alternatively composing these two dualities, the entire basic structure of Eqs. (55) can be recovered. To the best of our knowledge, the family (55), as a set of transformations, first appeared in [73], and, since then, in an amazing amount of other situations. Moreover, isolated elements of the family (55) had been present in the literature even before the paper [73]. The exhaustive list of these many situations is out of the present scope. Let us, however, mention a few paradigmatic ones.

First, the $q$-Fourier transform (that we will discuss in Section 4$)$, when applied to $(q, \alpha)$-stable distributions, involves $[20,74,75]$ the transformation $(53)$.

Second, the $q$-Fourier transform for $q<1$ can be obtained from that corresponding to $q>1$ by using the transformation [76]

$$
\frac{2}{q_{1}^{-}-1}=\frac{2}{1-q_{0}}+1
$$

which transforms the interval $[1,3)$ into the interval $(-\infty, 1]$ (see Fig. 5 ). This transformation is precisely the $(\alpha, m)=$ $(2,-1)$ element of family (54).

Third, elements of these families appear in what is sometimes called the $q$-triplet, which we address specifically in the next Subsection.

The sets (53) and (54) are illustrated in Figs. 6 and 7 respectively.

\subsection{The $q$-triplet}

It was heuristically conjectured in 2004 [77] that complex physical systems should exist with a set of relevant $q$ 's, namely $q_{\text {sensitivity }}<1, q_{\text {relaxation }}>1$ and $q_{\text {stationary state }}>1$. 
This set is currently referred to as the $q$-triangle, or the $q$ triplet. In the $B G$ limit, it should be $q_{\text {sensitivity }}=q_{\text {relaxation }}=$ $q_{\text {sensitivity }}=1$.

One year later, the $q$-triplet was indeed found in the solar wind [78] through the analysis of the magnetic-field data sent to Earth (NASA Goddard Space Flight Center) by the spacecraft Voyager 1, at the time in the distant heliosphere. The observations led to the following values:

$$
\begin{aligned}
q_{\text {sensitivity }} & =-0.6 \pm 0.2, \\
q_{\text {relaxation }} & =3.8 \pm 0.3, \\
q_{\text {stationary state }} & =1.75 \pm 0.06 .
\end{aligned}
$$

The most precise $q$ being $q_{\text {stationary state }}$, it seems reasonable to fix it at its nominal value, i.e.,

$$
q_{\text {stationary state }}=7 / 4 \text {. }
$$

By heuristically adopting simple relations belonging to the set (53), we can conjecture [58]

$$
q_{\text {relaxation }}=2-\frac{1}{q_{\text {sensitivity }}},
$$

and

$$
q_{\text {stationary state }}=2-\frac{1}{q_{\text {relaxation }}} .
$$

Eqs. (62), (63) and (64) lead to

$$
\begin{aligned}
q_{\text {sensitivity }} & =-1 / 2, \\
q_{\text {relaxation }} & =4, \\
q_{\text {stationary state }} & =7 / 4,
\end{aligned}
$$

which, within the error bars, are consistent with the observed values (59), (60) and (61). These assumptions are consistent with the following identification:

$$
\begin{aligned}
q_{\text {sensitivity }} & \equiv q_{m}^{+}, \\
q_{\text {relaxation }} & \equiv q_{m-\alpha}^{+}, \\
q_{\text {stationary state }} & \equiv q_{m-2 \alpha}^{+} .
\end{aligned}
$$

They are also consistent with the following identification

$$
\begin{aligned}
q_{\text {sensitivity }} & \equiv q_{m}^{-}, \\
q_{\text {relaxation }} & \equiv q_{m-\alpha}^{-}, \\
q_{\text {stationary state }} & \equiv q_{m-2 \alpha}^{-} .
\end{aligned}
$$

Although this kind of scenario is tempting, we have not yet achieved a deep understanding which would provide a physical justification about it. To make things even more intriguing, a last remarkable discovery [10, 79] deserves mention. Through the definition $\varepsilon \equiv 1-q$, we have

$$
\begin{aligned}
\varepsilon_{\text {sensitivity }} & \equiv 1-q_{\text {sensitivity }}=1-(-1 / 2)=3 / 2 \\
\varepsilon_{\text {relaxation }} & \equiv 1-q_{\text {relaxation }}=1-4=-3 \\
\varepsilon_{\text {stationary state }} & \equiv 1-q_{\text {stationary state }}=1-7 / 4=-3 / 4
\end{aligned}
$$

Amazingly enough, these values satisfy

$$
\begin{aligned}
\varepsilon_{\text {stationary state }} & =\frac{\varepsilon_{\text {sensitivity }}+\varepsilon_{\text {relaxation }}}{2}, \\
\varepsilon_{\text {sensitivity }} & =\sqrt{\varepsilon_{\text {stationary state }} \varepsilon_{\text {relaxation }}} \\
\varepsilon_{\text {relaxation }}^{-1} & =\frac{\varepsilon_{\text {sensitivity }}^{-1}+\varepsilon_{\text {stationary state }}^{-1}}{2}, .
\end{aligned}
$$

which are the arithmetic, geometric and harmonic means respectively! All these striking features suggest something like a deep symmetry, which eludes us. In any case, even if notoriously incomplete, the whole story was apparently considered quite stimulating by the organizers of the United Nations International Heliophysical Year 2007. Indeed, they prepared the poster shown in Fig. 8 for the exhibition organized during the launching of the IHY in Vienna.

One more interesting $q$-triplet is presently known, namely for the edge of chaos of the logistic map. It is given by

$$
\begin{aligned}
q_{\text {sensitivity }} & =0.244487701341282066198 \ldots, \\
q_{\text {relaxation }} & =2.249784109 \ldots \\
q_{\text {stationary state }} & =1.65 \pm 0.05
\end{aligned}
$$

We remind that, for this case, $q_{\text {entropy production }}=q_{\text {sensitivity }}$. See [80-83] for the value $(81)$, and $[84,85]$ for the value $(82)$. No simple relations seem to hold between these three numbers. We notice, however, that, for both $q$-triplets that have been presented in this Subsection, the following inequalities hold:

$$
q_{\text {sensitivity }} \leq 1 \leq q_{\text {stationary state }} \leq q_{\text {relaxation }}
$$

\section{4. $q$-GENERALIZING THE CENTRAL LIMIT THEOREM, OR WHY ARE THERE SO MANY $q$-GAUSSIAN-LIKE DISTRIBUTIONS IN NATURE?}

\subsection{Central limit theorems and $q$-Fourier transforms}

The Central Limit Theorem (CLT) with its Gaussian attractors (in the space of probability distributions) constitutes one of the main pieces mathematically grounding important parts of $B G$ statistical mechanics.

For instance, if we extremize $S_{B G}=-k \int_{-\infty}^{\infty} d x p(x) \ln p(x)$ with the constraints $\int_{-\infty}^{\infty} d x p(x)=1,\langle x\rangle \equiv \int_{-\infty}^{\infty} d x x p(x)=0$ and $\left\langle x^{2}\right\rangle \equiv \int_{-\infty}^{\infty} d x x^{2} p(x)=\sigma^{2}$, we straightforwardly obtain the following equilibrium distribution:

$$
p_{B G}(x)=\frac{e^{-\beta x^{2}}}{\int_{-\infty}^{\infty} d x e^{-\beta x^{2}}},
$$

i.e., a Gaussian, where the Lagrange parameter $\beta>0$ is determined through the $\sigma^{2}$-constraint.

On a different vein - still within the realm of $B G$ statistical mechanics - , the most basic free-particle Langevin equation (with additive noise), and its associated Fokker-Planck equation, provide, for all times and positions, a Gaussian distribution.

As a third connection, one might argue that the velocity distribution of any particle of a system described by a classical $N$-particle Hamiltonian with kinetic energy and (short-range) two-body interactions is the Maxwellian distribution, i.e., a Gaussian. Now, this comes out from $B G$ statistics. So, in what sense may we consider that the CLT enters? In the sense that Maxwellian distributions are indeed ubiquitously observed in nature, and this happens because, for not too strong perturbations, Gaussians are attractors.

Since $S_{q}$ generalizes $S_{B G}$, one expects the just mentioned connections (and similar ones) to be appropriately $q$ generalized. So should also be with the $C L T$ itself (see [86] 


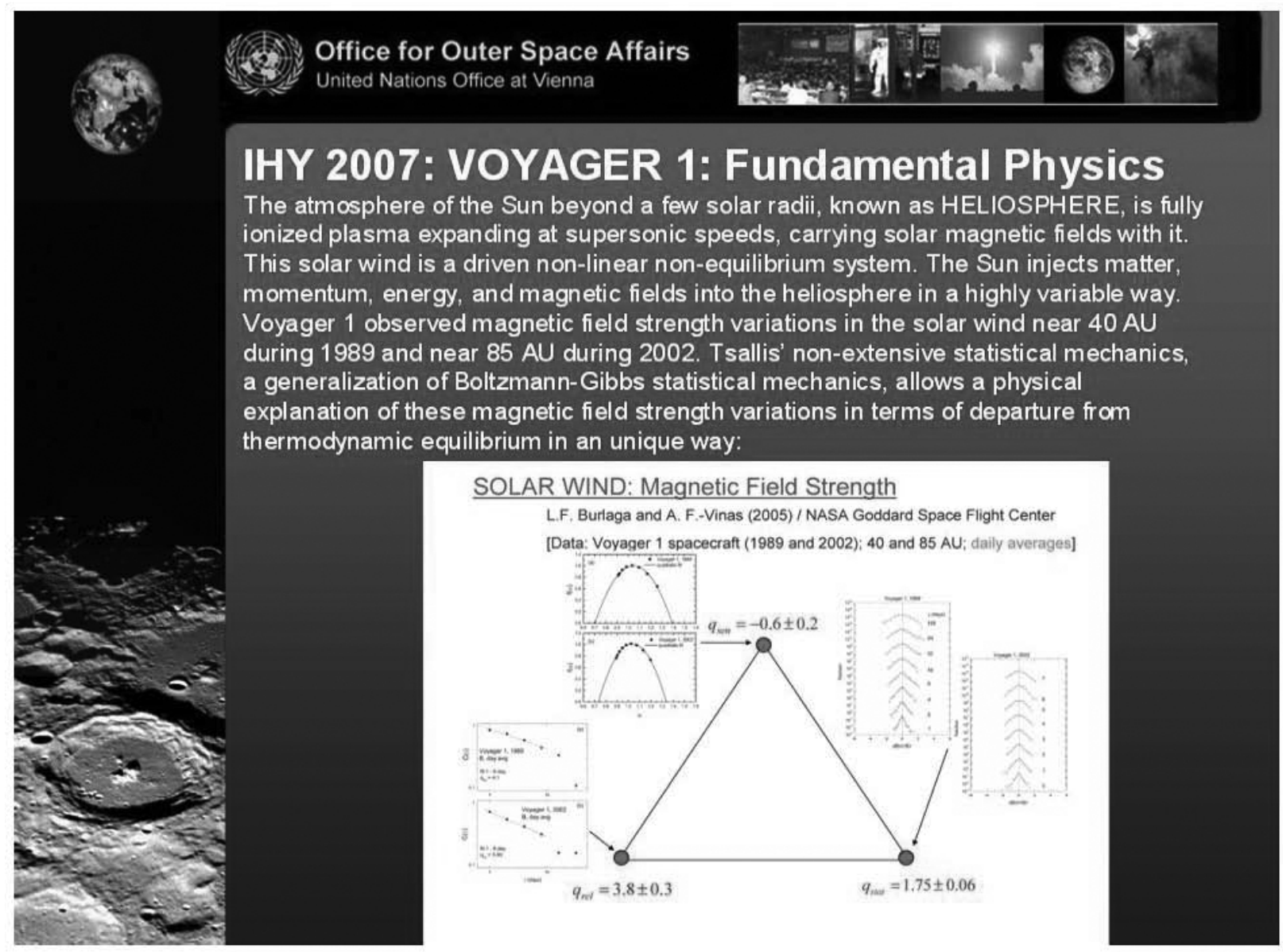

FIG. 8: Poster prepared by the organizers of the Opening Ceremony of the International Heliophysical Year 2007 for its accompanying exhibition, the 27 February 2007 in Vienna.

and references therein for the reasons which enabled conjecturing this $q$-generalization).

We do not intend in the present review to be exhaustive with regard to this rich subject. Let us nevertheless remind that the extremization of $S_{q}$ under essentially the same constraints as before (i.e., normalization, symmetry, and finiteness of the width of the distribution) yields, as the stationarystate distribution, the following $q$-Gaussian:

$$
p_{q}(x)=\frac{e_{q}^{-\beta x^{2}}}{\int_{-\infty}^{\infty} d x e_{q}^{-\beta x^{2}}} \quad(q<3 ; \beta>0) .
$$

Let us now briefly review the standard CLT as well as the occasionally called Lévy-Gnedenko theorem. If we have $N$ equal independent (or quasi-independent in an appropriate manner) random variables $x_{i}(i=1,2, \ldots, N)$, their sum $X_{N} \equiv \sum_{i=1}^{N} x_{i}$ converges (after appropriate centering and scaling with $N$ ) onto a Gaussian distribution in the $N \rightarrow \infty$ limit if the variance of the elementary distribution is finite. Such is the case, for instance, for $q$-Gaussians with $q<5 / 3$. If the variance of the elementary distribution diverges (and the distribution asymptotically decays like a power-law), the sum $X_{N}$ converges, instead, onto an $\alpha$-stable Lévy distribution. Such is the case, for instance, for $q$-Gaussians with $5 / 3<q<3$. In this case, through the $|x| \rightarrow \infty$ asymptotics, the Lévy index $\alpha$ is related to the index $q$ through

$$
\alpha=\frac{3-q}{q-1} \quad(5 / 3<q<3) .
$$

The situation changes drastically if the hypothesis of (quasi-)independence is violated, i.e., if the $N$ variables are strongly correlated. In this case, one expects the attractor to be neither Gaussian nor $\alpha$-stable. If this correlation is of the type denoted as $q$-independence (1-independence being independence) [20], then the attractor of the sum $X_{N}$ is a $q$ Gaussian if a specific $q$-generalized variance is finite, and a $(q, \alpha)$-stable distribution if it diverges. These are the respective $q$-generalizations of Gaussians and Lévy distributions. See Fig. 9.

The proof of these $q$-generalized $C L T$ theorems is based on the so called $q$-Fourier transform, defined as follows [20, 74, 75, 88, 89]:

$$
F_{q}[f](\xi) \equiv \int d x e_{q}^{i x \xi} \otimes_{q} f(x)=\int d x e_{q}^{i x \xi[f(x)]^{q-1}} f(x)
$$

for $q \geq 1$ and $f(x) \geq 0$. It is also possible to define the $q$ Fourier transform for $q<0$. This can be done through the 


\begin{tabular}{|c|c|c|}
\hline & $q=1 \quad[$ independent $]$ & $q \neq 1($ i.e., $Q \equiv 2 q-1 \neq 1)$ [globally correlated $]$ \\
\hline $\begin{array}{l}\sigma_{Q}<\infty \\
(\alpha=2)\end{array}$ & $\begin{array}{l}\mathbb{F}(x)=\text { Gaussian } G(x), \\
\text { with same } \sigma_{1} \text { of } f(x)\end{array}$ & $\begin{aligned} & \mathbb{F}(x)=G_{q}(x) \equiv G_{\left(3 q_{1}-1\right) /\left(1+q_{1}\right)}(x), \text { with same } \sigma_{Q} \text { of } f(x) \\
& G_{q}(x) \sim \begin{cases}G(x) & \text { if }|x|<<x_{c}(q, 2) \\
f(x) \sim C_{q} /|x|^{2 /(q-1)} & \text { if }|x|>>x_{c}(q, 2),\end{cases} \\
& \text { with } \lim _{q \rightarrow 1} x_{c}(q, 2)=\infty\end{aligned}$ \\
\hline $\begin{array}{l}\sigma_{Q} \rightarrow \infty \\
(0<\alpha<2)\end{array}$ & $\begin{array}{l}F(x)=\text { Levy distribution } L_{\alpha}(x), \\
\text { with same }|x| \rightarrow \infty \text { behavior } \\
\mathrm{L}_{\alpha}(x) \sim\left\{\begin{array}{c}G(x) \\
\text { if }|x|<<x_{c}(1, \alpha) \\
f(x) \sim C_{\alpha} /|x|^{1+\alpha} \\
\text { if }|x|>>x_{c}(1, \alpha)\end{array}\right. \\
\text { with } \lim _{\alpha \rightarrow 2} x_{c}(1, \alpha)=\infty\end{array}$ & $\begin{array}{c}\mathbb{F}(x)=L_{q, \alpha}, \text { with same }|x| \rightarrow \infty \text { asymptotic behavior } \\
L_{q, \alpha} \sim\left\{\begin{array}{cc}G_{\frac{2(1-q)-\alpha(1+q)}{2(1-q)-\alpha(3-q)}, \alpha}(x) \sim C_{q, \alpha}^{*} /|x|^{\frac{2(1-q)-\alpha(3-q)}{2(1-q)}} \\
(\text { intermediate regime }) \\
G_{\frac{2 \alpha q-\alpha+3}{\alpha+1}, 2}(x) \sim C_{q, \alpha}^{L} /|x|^{(1+\alpha) /(1+\alpha q-\alpha)} \\
(\text { distant regime })\end{array}\right.\end{array}$ \\
\hline
\end{tabular}

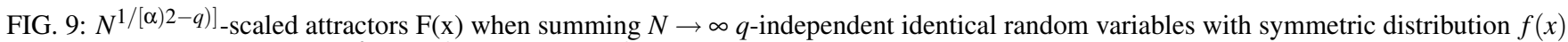
with $Q$-variance $\sigma_{Q} \equiv \int_{-\infty}^{\infty} d x x^{2}[f(x)]^{Q} / \int_{-\infty}^{\infty} d x[f(x)]^{Q}\left(Q \equiv 2 q-1 ; q_{1}=(1+q) /(3-q) ; q \geq 1\right)$. Top left: The attractor is the Gaussian sharing with $f(x)$ the same variance $\sigma_{1}$ (standard CLT). Bottom left: The attractor is the $\alpha$-stable Lévy distribution which shares with $f(x)$ the same asymptotic behavior, i.e., the coefficient $C_{\alpha}$ (Lévy-Gnedenko CLT, or $\alpha$-generalization of the standard CLT). Top right: The attractor is the $q$-Gaussian which shares with $f(x)$ the same $(2 q-1)$-variance, i.e., the coefficient $C_{q}$ ( $q$-generalization of the standard CLT, or $q$-CLT). Bottom right: The attractor is the $(q, \alpha)$-stable distribution which shares with $f(x)$ the same asymptotic behavior, i.e., the coefficient $C_{q . \alpha}^{L}$ ( $q$-generalization of the Lévy-Gnedenko CLT and $\alpha$-generalization of the $q$-CLT). The case $\alpha<2$, for both $q=1$ and $q \neq 1$ (more precisely $q>1$ ), further demands specific asymptotics for the attractors to be those indicated; essentially the divergent $q$-variance must be due to fat tails of the power-law class, except for possible logarithmic corrections (for the $q=1$ case see, for instance, [87] and references therein).

transformation (58) [76]. The $q$-Fourier transform (which is nonlinear if $q \neq 1$ ) has a remarkable property, namely to be closed within the family of $q$-Gaussians. More precisely, the $q$-Fourier transform of a (normalized) $q$-Gaussian, as given by Eq. (85), is another member of the $q$-Gaussian family, namely one having its index given by

$$
q_{1}=\frac{1+q}{3-q},
$$

which admits $q=1$ as a fixed point (corresponding to the standard Fourier tranform). It can be rewritten as follows:

$$
\frac{2}{1-q_{1}}=\frac{2}{1-q}+1
$$

which, through Eq. (40), immediately allows for the identification $\left(q, q_{1}\right) \equiv\left(q_{0}, q_{1}^{+}\right)$for $\alpha=2$.

This transformation, together with the corresponding one for $\beta$, is straightforwardly reversible. However, for a density $f(x)$ not belonging to the family of $q$-Gaussians, the problem can be more complex. Hilhorst has introduced [90] two interesting and paradigmatic examples which illustrate the difficulty. One of them is presented in his article [91] in this same volume. We shall here discuss his other example, which we present in what follows. Let us define the probability density

$$
f(x, a) \equiv\left\{\begin{array}{cl}
\frac{\sqrt{2}}{\pi} \frac{(1-a|x|)^{2}}{\left[(1-a|x|)^{2}+\frac{1}{2} x^{2}\right]^{2}} & \text { if }|x|<1 / a \\
0 & \text { otherwise }
\end{array}\right.
$$

with $a \geq 0$. We verify that $f(x, a)$ is normalized, $\forall a$, i.e.,

$$
\int_{-1 / a}^{1 / a} d x f(x, a)=1, \quad \forall a .
$$

We also verify that $f(x, 0)$ is precisely but a $(3 / 2)$-Gaussian, namely

$$
f(x, 0)=\frac{\sqrt{2}}{\pi} \frac{1}{\left(1+\frac{1}{2} x^{2}\right)^{2}} .
$$

See Fig. 10 for typical examples of $f(x, a)$. Its $q$-Fourier transform for $q \geq 1$ is given by

$$
F_{q}[f(x, a)](\xi)=\int_{-\infty}^{\infty} d x \frac{f(x, a)}{\left\{1-(q-1) i \xi x[f(x, a)]^{q-1}\right\}^{\frac{1}{q-1}}} .
$$




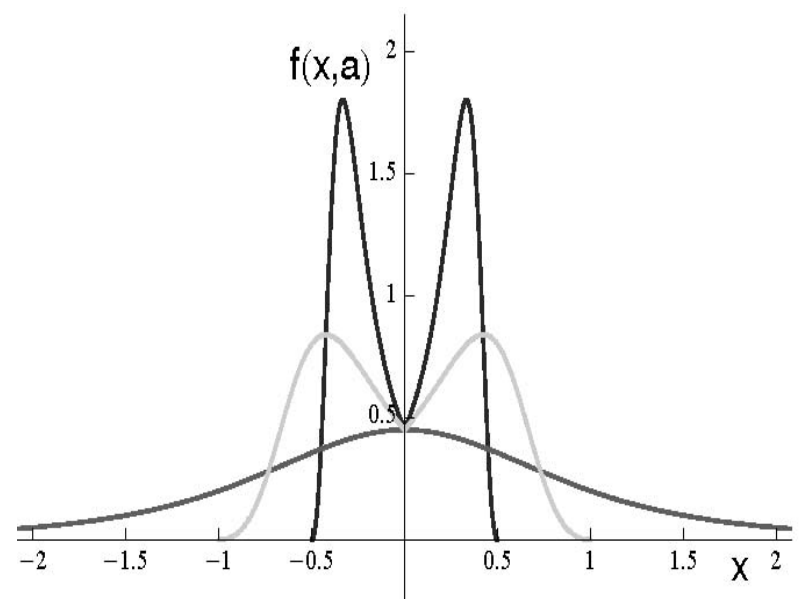

FIG. 10: The distribution $f(x, a)$ for typical values of $a: a=2$ (blue), $a=1$ (green), and $a=0$ (red). From [92].

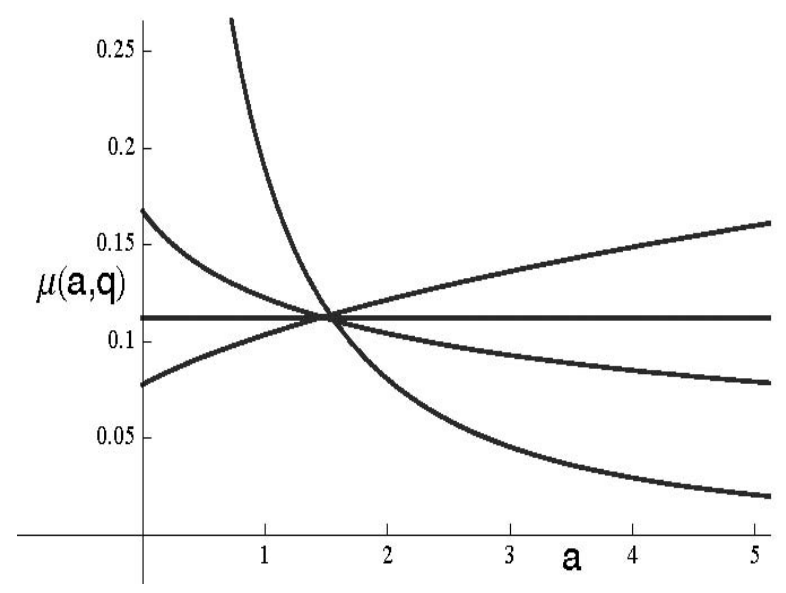

FIG. 11: The $a$-dependence of $\mu(a, q)$ for typical values of $q$. From top to bottom on the right side: $q=1.6,1.5,1.4,1$. From [92].

Replacing herein Eq. (90) we obtain

$$
\begin{aligned}
& F_{q}[f(x, a)](\xi)=\int_{-1 / a}^{1 / a} d x \\
& \frac{\sqrt{2}}{\pi} \frac{(1-a|x|)^{2}}{\left[(1-a|x|)^{2}+x^{2} / 2\right]^{2}} \\
& \overline{\left\{1-(q-1) i \xi x\left[\frac{\sqrt{2}}{\pi} \frac{(1-a|x|)^{2}}{\left[(1-a|x|)^{2}+x^{2} / 2\right]^{2}}\right]^{q-1}\right\}^{\frac{1}{q-1}}}
\end{aligned}
$$

It can be verified that $F_{q}[f(x, a)](\xi)$ depends on $a$ for any $q \neq$ $3 / 2$. But, $F_{3 / 2}[f(x, a)](\xi)$ does not depend on $a$. Indeed, it is given by

$$
F_{3 / 2}[f(x, a)](\xi)=\frac{1}{\left[1+\frac{1}{4 \pi \sqrt{2}} \xi^{2}\right]^{3 / 2}}
$$

where we have used the variable changement $y=\frac{x}{1-a|x|}$. In other words, this $q$-Fourier integral transform has, not one, but an entire family of functions (parameterized by the real

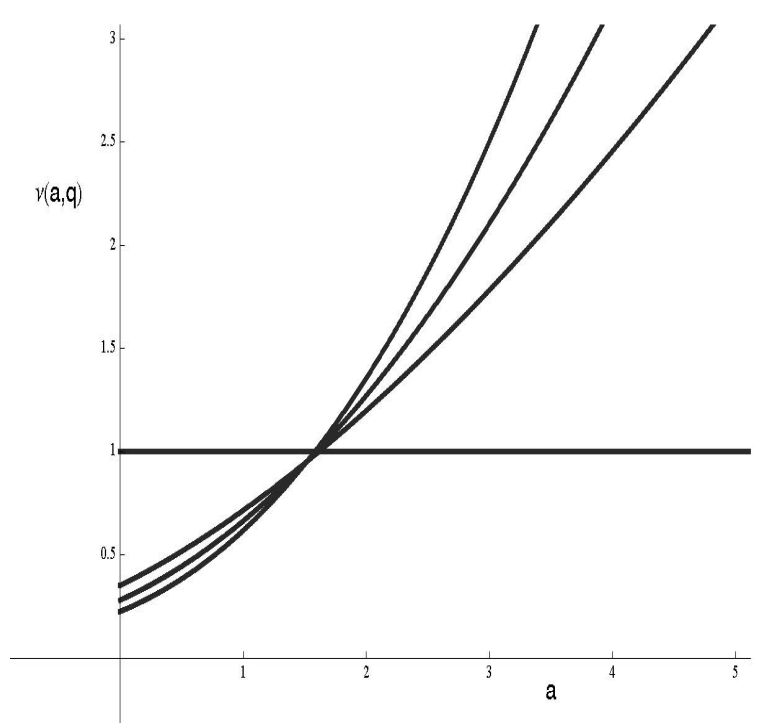

FIG. 12: The $a$-dependence of $v(a, q)$ for typical values of $q$. From top to bottom on the right side: $q=1.6,1.5,1.4,1$. From [92].

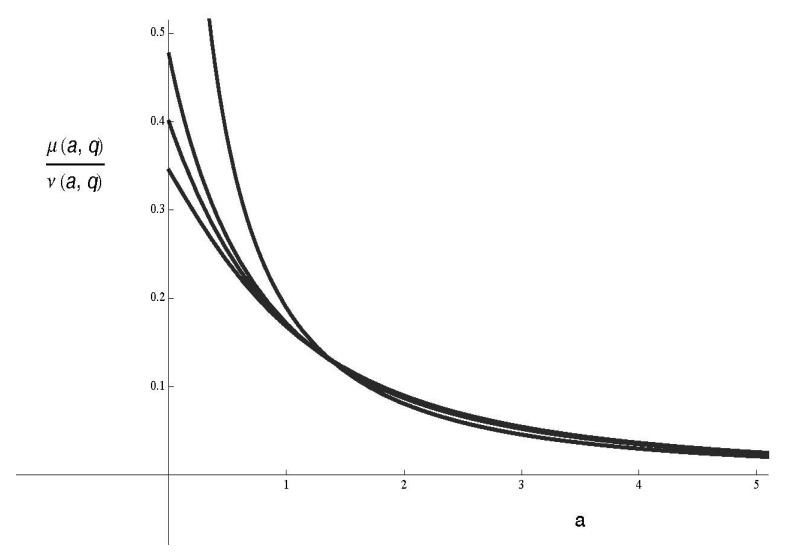

FIG. 13: The $a$-dependence of $\left\langle x^{2}\right\rangle_{2 q-1}=\mu(a, q) / v(a, q)$ for typical values of $q$. From top to bottom on the left side: $q=1,1.4,1.5,1.6$. From [92].

number $a$ ) of pre-images. Hence, unless we add some supplementary information, $F_{3 / 2}[f(x, a)](\xi)$ is not invertible. Let us now discuss this delicate point related to the nonlinearity of the generic $q$-Fourier transform, a point which evaporates for $q=1$ since the standard Fourier tranform is a linear one.

At the present stage, let us focus, not on the generic problem of finding which is the general form for $f(x)$ whose $3 / 2$ Fourier transform is given by Eq. (95), but on a less ambitious one, namely: Knowing that we are dealing within the family $\{f(x, a)\}$, which is the specific value of the parameter $a$ that we are interested in? We show next that this question can be univoquely answered with some simple supplementary information. Let us illustrate now how. 
We first define the following quantities [23]:

$$
\mu_{2 q-1} \equiv \int_{-1 / a}^{1 / a} d x x^{2}[f(x, a)]^{2 q-1} \equiv \mu(a, q) \quad[\mu(0,1)=2],
$$

and

$$
v_{2 q-1} \equiv \int_{-1 / a}^{1 / a} d x[f(x, a)]^{2 q-1} \equiv v(a, q) \quad[v(a, 1)=1],
$$

which are illustrated in Figs. 11 and 12 respectively. The $(2 q-1)$-variance is given by

$$
\left\langle x^{2}\right\rangle_{2 q-1} \equiv \frac{\int_{-1 / a}^{1 / a} d x x^{2}[f(x, a)]^{2 q-1}}{\int_{-1 / a}^{1 / a} d x[f(x, a)]^{2 q-1}}=\frac{\mu(a, q)}{v(a, q)} .
$$

This quantity corresponds to the standard (linear) variance, but with the escort distribution $[f(x, a)]^{2 q-1} / \int_{-1 / a}^{1 / a} d x^{\prime}\left[f\left(x^{\prime}, a\right)\right]^{2 q-1}$, instead of the original one $f(x, a)$. It is illustrated in Fig. 13.

To fully understand the implications of these results, let us remind that successive moments with appropriate escort distributions are directly related with the successive derivatives of the $q$-Fourier transform of $f(x, a)$ [23]. Since $f(x, a)$ is an even function, all odd moments, as well as all odd derivatives of $\left.F_{q}[f(x, a)](\xi)\right|_{\xi=0}$, vanish. All even such derivatives are finite, in particular $\left.\mu_{2 q-1} \propto \frac{d^{2} F_{q}[f(x, a)](\xi)}{d \xi^{2}}\right|_{\xi=0}$. We see in Fig. 11 that, for all $q \neq 3 / 2, \mu_{2 q-1}$ monotonically depends on $a$. Therefore, for all such values of $q$ and within the family $\{f(x, a)\}$, the $q$-Fourier integral transform is invertible, even being nonlinear. However, not so for $q=3 / 2$. Consistently, $\mu(a, 3 / 2)$ is a constant. In this particular case, further information is needed. This information can be the value of $v(a, 3 / 2)$, which, as we verify in Fig. 12, monotonically depends on $a$. In other words, the integral tranform $F_{3 / 2}$ together with the knowledge of say $v(a, 3 / 2)$, becomes an invertible operation within the family $\{f(x, a)\}$.

An analogy can be done at this point. As we all know, Newton's equation $m \frac{d^{2} x(t)}{d t^{2}}=F(x)$ does not provide a single solution but a family of solutions. To have a unique solution we need to provide further information, namely $x(0)$ and $\dot{x}(0)$. An important difference, however, between this problem and the generic invertibility of the $q$-Fourier transform is that, for the nonlinear integral transform, we do not know the general form of its inverse. Would we know it, the problem would in principle be completely solvable through the use of supplementary information (such as the value of $n u$ for the above illustration) which would uniquely determine the specific density within the general form. As we see, the general problem still remains open. But if we happen to know within which specific family of densities we are working (say $q$-Gaussians, or say the family $\{f(x, a)\}$ ), uniqueness of the inverse is basically guaranteed.

All the above discussion about invertibility is of course relevant for the domain of validity of the $q$-generalized central limit theorem. At the present moment, its proof [20] fully applies only within the class of densities for which invertibility is guaranteed. This class is very vast; however, as we have seen, it does not include all possible densities. An alternative $q$-generalized $C L T$ can be seen in [93].

\subsection{Fittings can be extremely useful ... when done carefully!}

In this section we compare three analytic forms which are frequently used in the context of complex systems whenever fat tails emerge. These forms are the q-exponential, the stretched exponential, and the Mittag-Leffler function. All three recover the exponential function as a limiting instance.

Let us remind that the q-exponential function is defined, for $x \geq 0$ and $q \geq 1$, as follows:

$$
e_{q}^{-x} \equiv \frac{1}{[1+(q-1) x]^{\frac{1}{q-1}}} \quad\left(e_{1}^{-x}=e^{-x}\right)
$$

hence, for $\beta \geq 0$,

$$
e_{q}^{-\beta x} \equiv \frac{1}{[1+(q-1) \beta x]^{\frac{1}{q-1}}} \quad\left(e_{1}^{-\beta x}=e^{-\beta x}\right)
$$

We immediately verify that

$$
e_{q}^{-\beta x} \sim 1-\beta x \quad(x \rightarrow 0 ; \forall q \geq 1),
$$

and that

$$
e_{q}^{-\beta x} \sim \frac{1}{[(q-1) \beta]^{\frac{1}{q-1}}} \frac{1}{x^{\frac{1}{q-1}}} \propto \frac{1}{x^{\frac{1}{q-1}}} \quad(x \rightarrow \infty ; \forall q>1) .
$$

Let us finally mention that the function (100) is the solution of

$$
\frac{d y}{d x}=-\beta y^{q}
$$

with $y(0)=1$.

Let us consider now, for comparison, the stretched exponential function, defined, for $0<\alpha \leq 1$, as follows:

$$
\mathcal{E}_{\alpha}(x) \equiv e^{\operatorname{sign}(x)|x|^{\alpha}} \quad\left(\mathcal{E}_{1}(x)=e^{x}\right),
$$

hence, for $x \geq 0$ and $\gamma \geq 0$,

$$
\mathcal{E}_{\alpha}\left(-\gamma^{\frac{1}{\alpha}} x\right) \equiv e^{-\gamma x^{\alpha}} \quad\left(\mathcal{E}_{1}(-\gamma x)=e^{-\gamma x}\right)
$$

We immediately verify that

$$
\mathcal{E}_{\alpha}\left(-\gamma^{\frac{1}{\alpha}} x\right) \sim 1-\gamma x^{\alpha} \quad(x \rightarrow 0 ; 0<\alpha \leq 1),
$$

and that

$$
\mathcal{E}_{\alpha}\left(-\gamma^{\frac{1}{\alpha}} x\right)=e^{-\gamma x^{\alpha}} \quad(x \rightarrow \infty ; 0<\alpha \leq 1) .
$$

The function (104) is the solution of

$$
\frac{d y}{d\left[\operatorname{sign}(x)|x|^{\alpha}\right]}=-\gamma y
$$

with $y(0)=1$.

Let us now add, within this comparison, the Mittag-Leffler function defined, for $0<\eta \leq 1$, as follows (see, for instance, [94-96]):

$$
E_{\eta}(x)=\sum_{n=0}^{\infty} \frac{x^{n}}{\Gamma(1+\eta n)} \quad\left(E_{1}(x)=e^{x}\right) .
$$


It follows that, for $x \geq 0$ and $\delta \geq 0$, we have

$$
E_{\eta}\left(-(\delta x)^{\eta}\right)=\sum_{n=0}^{\infty}(-1)^{n} \frac{(\delta x)^{\eta n}}{\Gamma(1+\eta n)}
$$

whose asymptotic behaviors are

$$
E_{\eta}\left(-(\delta x)^{\eta}\right) \sim 1-\frac{(\delta x)^{\eta}}{\Gamma(1+\eta)} \quad(x \rightarrow 0 ; 0<\eta \leq 1),
$$

and

$$
E_{\eta}\left(-(\delta x)^{\eta}\right) \sim \frac{1}{\Gamma(1-\eta)(\delta x)^{\eta}} \quad(x \rightarrow \infty ; 0<\eta<1) .
$$

Consequently, this function interpolates between the stretched exponential for small values of $x$ and the power-law for large values of $x$ (see Fig. 22 of [95]).

The function (110) satisfies

$$
\frac{d^{\eta} y}{d x^{\eta}}=-\delta^{\eta} y
$$

with $y(0)=1$, the Caputo fractional derivative (which differs from the Riemman-Liouville fractional derivative) being defined as follows:

$$
\frac{d^{\eta} y}{d x^{\eta}} \equiv \frac{1}{\Gamma(1-\eta)} \int_{0}^{x} d x^{\prime} \frac{y^{(1)}\left(x^{\prime}\right)}{\left(x-x^{\prime}\right)^{\eta}} \quad(0<\eta<1),
$$

where $y^{(1)}\left(x^{\prime}\right) \equiv \frac{d y\left(x^{\prime}\right)}{d x^{\prime}} ; \lim _{\eta \rightarrow 1} \frac{d^{\eta} y}{d x^{\eta}}=\frac{d y}{d x}$.

We see therefore that, if we have access to the $x \rightarrow 0$ and $x \rightarrow \infty$ asymptotic behaviors, $q$-exponentials $(q>1)$, stretched exponentials $(0<\alpha<1)$, and Mittag-Leffler functions $(0<\eta<1)$ are easily distinguishable among them. However, as illustrated in Figs. 14 and 15, their values can be very similar in the intermediate (non asymptotic) region. This fact illustrates a well known concept: careless fittings can be dangerous. They can be however extremely useful if done meticulously. They are, in any case, inescapable whenever the analysis of experimental or numerical results is concerned. Further interesting examples along similar lines can be seen in [91].

The three functions that we have compared here are particular cases of the function $y(x ; q, \alpha, \eta, B)$ which satisfies

$$
\frac{d^{\eta} y}{d\left(x^{\alpha}\right)^{\eta}}=-B y^{q}
$$

with $y(0 ; q, \alpha, \eta, B)=1$. The $q$-exponential corresponds to $y(x ; q, 1,1, \beta)$; the stretched exponential corresponds to $y(x ; 1, \alpha, 1, \gamma)$; and the Mittag-Leffler function corresponds to $y\left(x ; 1,1, \eta, \delta^{\eta}\right)$. Clearly, at the present time no analytical expression exists for such a general function $y(x ; q, \alpha, \eta, B)$.

\section{3. $q$-Gaussian-like distributions in nature and elsewhere}

The $q$-generalized CLT, with its $q$-Gaussian attractors in the space of probability densities, applies for correlations of the $q$-independent type. There is strong evidence that this type of correlations is deeply related to strict or asymptotic
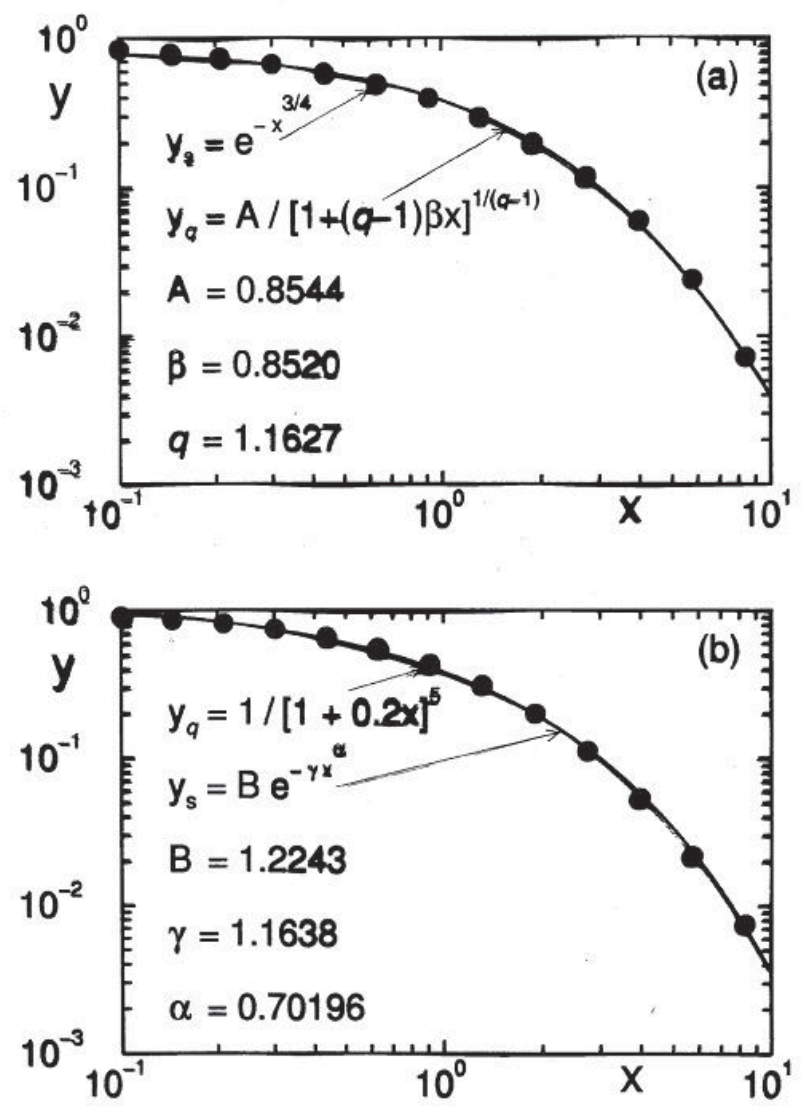

FIG. 14: Comparing $q$-exponentials with stretched exponentials in $\log -\log$ scale (which is the most frequent representation of such functions). (a) The dots have been generated with the stretched exponential function $y=e^{-x^{3 / 4}}$, their size has been enlarged in order to mimic experimental error bars, and they have been fitted, within the interval $\left[10^{-1}, 10\right]$, with the $q$-exponential $y=A e_{q}^{-\beta x}$. (b) The dots have been generated with the $q$-exponential function $y=e_{1.2}^{-x}$ , and they have been fitted, within the interval $\left[10^{-1}, 10\right]$, with the stretched exponential $y=B e^{-\gamma x^{\alpha}}$. As we verify in these two typical examples, these fitting functions are numerically indistinguishable within the intermediate interval that we have considered here; only high-precision numerical knowledge of the values of the "experimental" dots within the $x \rightarrow 0$ and $x \rightarrow \infty$ asymptotic regions could indicate one or the other, ... or a different one!

scale-invariance (which might well be necessary for $q$ independence, although surely not sufficient). After decades of studies focusing on fractals, it is by now well established that scale-invariance is ubiquitous in natural, artificial and even social systems. Consequently, we should expect the emergence of $q$-Gaussians quite often. This is indeed what happens, as we shall illustrate next (needless to say that only within the error bars corresponding to each case).

(i) As predicted by Lutz in 2003 [61], the velocity distribution of cold atoms in dissipative optical lattices has been seen in 2006 to be $q$-Gaussian [97]. This has been verified both with quantum Monte Carlo methods, as well as in the laboratory with $C s$ atoms. 

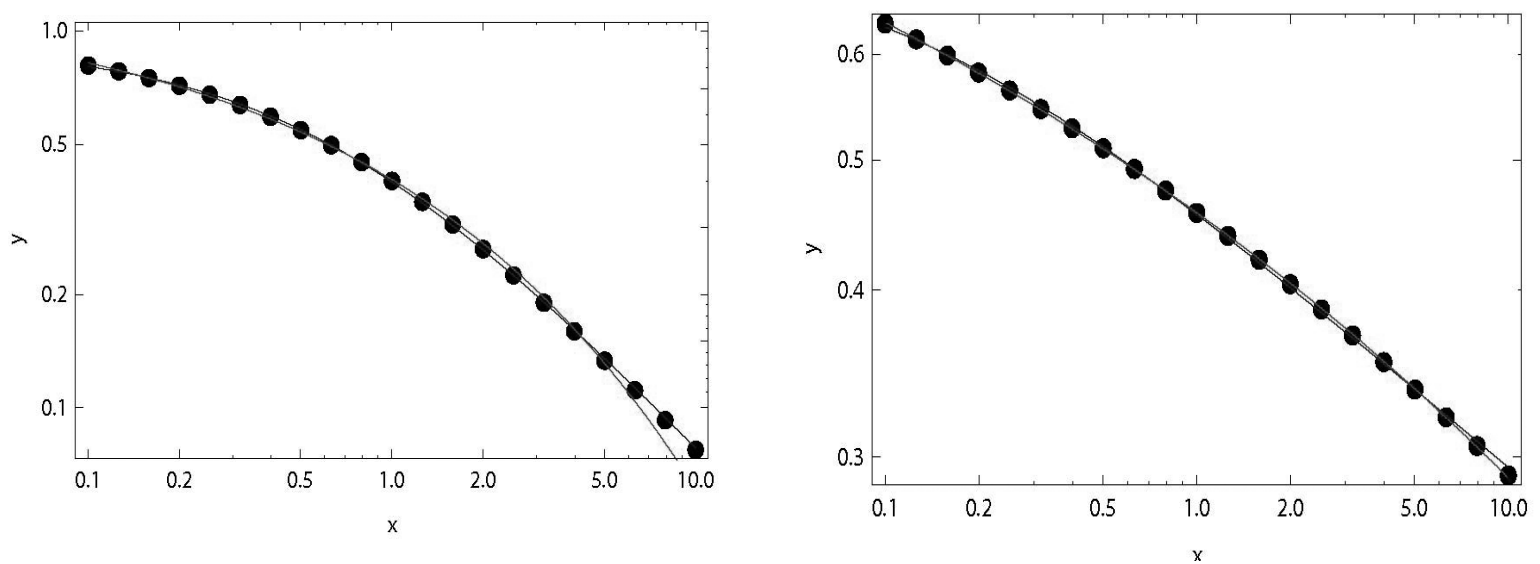

FIG. 15: The dots have been generated with the Mittag-Leffler function $y=E_{\eta}\left(-(\delta x)^{\eta}\right)$. The upper (lower) curve at the right side is the $q$-Gaussian $y=A e_{q}^{-\beta x}$ (stretched exponential $y=B e^{-\gamma x^{\alpha}}$ ) which best fits the present discrete set of 21 values of this Mittag-Leffler function. Left: $(\eta, \delta)=(0.7,1),(A, q, \beta)=(0.915335,2.15899,1.40801)$ and $(B, \alpha, \gamma)=(1.26496,0.423769,1.14411)$. Right: $(\eta, \delta)=(0.3,1)$, $(A, q, \beta)=(0.708294,6.06448,1.65478)$ and $(B, \alpha, \gamma)=(1.45013,0.144472,1.15538)$. We verify that, within this limited intermediate region and with the precision indicated by the size of the dots, the three functions are nearly indistinguishable. However, the detailed study of their $x \rightarrow 0$ and $x \rightarrow \infty$ asymptotic behaviors would allow for discrimination among them.

(ii) The distribution of velocities in quasi-two dimensional dusty plasma has been detected to be $q$-Gaussian $[98,99]$.

(iii) Single ions in radio frequency traps interacting with a classical buffer gas (e.g., ${ }^{136} \mathrm{Ba}^{+}$ion cooled at $300 \mathrm{~K}$ ) exhibit $q$-Gaussian distributions [100].

(iv) The distribution of velocities of cells of Hydra viridissima in solution has been shown to be $q$-Gaussian [101].

(v) Distribution of velocities of the defects in the so-called defect turbulence have been interpreted as $q$-Gaussians [102]

(vi) Distributions corresponding to fluctuations of the magnetic field in the plasma within the solar wind in the distant heliosphere and the heliosheath, as detected through data received from the Voyager 1 have been interpreted as $q$-Gaussians [78, 103-107].

(vii) Computational distributions of velocities in silo drainage of granular matter have been interpreted as $q$ Gaussians [108, 109].

(viii) Distributions of sums of velocities along the trajectories of the particles in a large class of initial conditions leading to the so-called quasi-stationary states of the HMF model (many-body Hamiltonian system describing an inertial classical $X Y$ ferromagnet with long-range two-body interactions) gradually approach $q$-Gaussians [110-112]. Let us mention at this point that if, instead of these time averages, we do ensemble averages, we obtain other types of distributions [113, 114].

(ix) Distributions of sums of velocities along the trajectories of the particles at the edge of chaos of the Kuramoto model gradually approach $q$-Gaussians [115]. (x) The distributions of sums of successive iterates in the close neighborhood of the edge of chaos in unimodal dissipative maps exhibit slight oscillations (possibly log-periodic) on top of $q$-Gaussians [84, 85, 116].

(xi) Return distributions corresponding to earthquakes using data from the World and Southern California catalogs have been interpreted as $q$-Gaussians [117].

(xii) Return distributions of fluctuations associated with self-organized criticality in the Ehrenfest's dog-flea model are consistent with $q$-Gaussians [118].

(xiii) Distributions of the increments of current fluctuations of charge disorder in arrays are interpreted as $q$-Gaussians [119].

(xiv) Financial return distributions in the New York Stock Exchange, NASDAQ and elsewhere are routinely interpreted as $q$-Gaussians [120-130].

(xv) Distributions of postural sway for young and old people are interpreted as $q$-Gaussians [131].

The $q$-Gaussian function is of course a $q$-exponential of a squared variable. The $q$-CLT only addresses $q$-Gaussians, not $q$-exponentials in general. However, it is clear that the detection of a $q$-exponential in some central property of a system opens the door for possibly finding $q$-Gaussians in some other important property. There are dozens of such situations that have been reported in the literature (see, for instance, [10]). We would like to add here a very recent one which concerns spin-glasses. This system was for many years awaited to exhibit connections with nonextensive statistics due to its notorious nonergodicity and complex structure in phase 
space. Very recently this finally occurred in the experimentally observed relaxation curves [132] of paradigmatic spinglasses such as $C u_{0.95} M n_{0.05}, C u_{0.90} M n_{0.10}, C u_{0.84} M n_{0.16}$, $C u_{0.65} M n_{0.35}$, and $A u_{0.86} F e_{0.14}$.

\section{CONCLUSIONS}

The magnificent theory of Boltzmann and Gibbs has, as any other intellectual construct, limits of validity. Outside these limits a vast world exists in nature as well as in artificial systems. Some - apparently many - classes of systems of this vast world are adequately addressed by nonextensive statistical mechanics, proposed over twenty years ago [9] as a generalization of the standard theory. The complexity of this extended theory certainly is much higher than that of the BG statistical mechanics. A central feature which dramatically reflects this complexity is the fact that only one value of the index $q$ is to be used for the BG theory, namely $q=1$, whereas an entire set of inter-related values for such indices emerges outside it (see, for instance, Figs. 6 and 7). Another feature which also reflects this complexity is the fact that the involved mathematics is definitively nonlinear, whereas that of the BG theory is, in many aspects, a linear one. Under such circumstances, one could expect to have an extremely slow technical and epistemological progress. The intensive and world-wide developments that we have witnessed during the last two decades neatly show that it has not been so. Indeed, an amazingly large field of knowledge is now available, demanding for further analytical, experimental, and computational efforts to advance. Sem pressa e sem pausa... (Without hurry and without stops...)

\section{Acknowledgements}

The idea of comparing, together with the $q$-exponential and the stretched exponential functions, the Mittag-Leffler function emerged during an interesting conversation with Ryszard Kutner, to whom I am indebted. Along this line, I also thank M.J. Jauregui Rodriguez for the construction of Fig. 15. I thank as well G. Ruiz for the construction of Figs. 6 and 7. I am indebted to H.J. Hilhorst for authorizing me to present his unpublished example, which enables the analysis of the invertibility of the $q$-Fourier transform out from the family of the $q$-Gaussians, although within a specific larger family of densities. I am also indebted to him, as well as to S. Umarov and E.M.F. Curado, for long and fruitful discussions, always on the subject of the nontrivial difficulties related with the $q$-Fourier transform possible invertibility. My thanks also go to R. Kutner and E.K. Lenzi for useful discussions about fractional derivatives. I am deeply grateful to R.S. Mendes, L.R. Evangelista, L.C. Malacarne and E.K. Lenzi for their efficient organization of, and their delightfully warm hospitality at, NEXT2008 - International Conference on Nonextensive Statistical Mechanics: Foundations and Applications, held in Iguassu-Brazil in 27-31 October 2008. Partial financial support by $\mathrm{CNPq}$ and FAPERJ (Brazilian agencies) is gratefully acknowledged.
[1] M. Planck, Uber irreversible Strahlugsforgange, Ann d. Phys. (4) (1900) 1, S. 69-122 (see [8]).

[2] M. Planck, Verhandlungen der Deutschen Physikalischen Gessellschaft 2, 202 and 237 (1900) (English translation: D. ter Haar, S. G. Brush, Planck's Original Papers in Quantum Physics (Taylor and Francis, London, 1972)].

[3] L.B. Okun, The fundamental constants of physics, Sov. Phys. Usp. 34, 818 (1991).

[4] G. Cohen-Tannoudji, Les Constantes Universelles, Collection Pluriel (Hachette, Paris, 1998).

[5] C. Tsallis and S. Abe, Advancing Faddeev: Math can deepen Physics understanding, Physics Today 51, 114 (1998).

[6] L. Okun, Cube or hypercube of natural units?, hepth/0112339 (2001).

[7] M.J. Duff, L.B. Okun and G. Veneziano, Trialogue on the number of fundamental constants, JHEP03 (2002) 023 [physics/0110060].

[8] M.J. Duff, Comment on time-variation of fundamental constants, hep-th/0208093 (2004).

[9] C. Tsallis, Possible generalization of Boltzmann-Gibbs statistics, J. Stat. Phys. 52, 479-487 (1988) [The proposal first came to my mind in August 1985, during a scientific event in Mexico City, and was further advanced in August 1987, during and immediately after a scientific event in Maceio-Brazil. But it was published only in 1988 , particularly stimulated by the fruitful discussions held on the subject with E.M.F. Curado and H.J. Herrmann during the Maceio event].

[10] C. Tsallis, Introduction to Nonextensive Statistical Mechanics - Approaching a Complex World (Springer, New York, 2009).

[11] A regularly updated Bibliography can be seen at http://tsallis.cat.cbpf.br/biblio.htm
[12] C. Tsallis, R.S. Mendes and A.R. Plastino, The role of constraints within generalized nonextensive statistics, Physica A 261, 534 (1998).

[13] E.M.F. Curado and C. Tsallis, Generalized statistical mechanics: connection with thermodynamics, J. Phys. A 24, L69 (1991); Corrigenda: 24, 3187 (1991) and 25, 1019 (1992).

[14] S. Abe, Instability of q-averages in nonextensive statistical mechanics, Europhys. Lett. 84, 60006 (2008).

[15] R. Hanel, S. Thurner and C. Tsallis, On the robustness of $q$ expectation values and Rényi entropy, Europhysics Letters 85, 20005 (2009).

[16] R. Hanel and S. Thurner, Stability criteria for q-expectation values, Phys. Lett. A 373, 1415 (2009).

[17] S. Abe and G.B. Bagci, Necessity of q-expectation value in nonextensive statistical mechanics, Phys. Rev. E 71, 016139 (2005).

[18] S. Abe, Why q-expectation values must be used in nonextensive statistical mechanics, Astrophys. Space Sci. 305, 241245 (2006).

[19] W. Thistleton, J.A. Marsh, K. Nelson and C. Tsallis, Generalized Box-Muller method for generating $q$-Gaussian random deviates, IEEE Transactions on Information Theory 53, 4805 (2007).

[20] S. Umarov, C. Tsallis and S. Steinberg, On a q-central limit theorem consistent with nonextensive statistical mechanics, Milan J. Math. 76, 307 (2008).

[21] J.-F. Bercher, Tsallis distribution as a standard maximum entropy solution with 'tail' constraint, Phys. Lett. A 372, 5657 (2008).

[22] J. Naudts, The q-exponential family in statistical physics, Central Eur. J. Phys. 7, 405 (2009). 
[23] C. Tsallis, A.R. Plastino and R.F. Alvarez-Estrada, Escort mean values and the characterization of power-law-decaying probability densities, J. Math. Phys. 50, 043303 (2009).

[24] V. Schwammle, F.D. Nobre and E.M.F. Curado, Consequences of the H-theorem from nonlinear Fokker-Planck equations, Phys. Rev. E 76, 041123 (2007).

[25] V. Schwammle, E.M.F. Curado and F.D. Nobre, A general nonlinear Fokker-Planck equation and its associated entropy, Eur. Phys. J. B 58, 159 (2007).

[26] S. Abe, Generalized molecular chaos hypothesis and $\mathrm{H}$ theorem: Problem of constraints and amendment of nonextensive statistical mechanics, Phys. Rev. E 79, 041116 (2009).

[27] G.L. Ferri, S. Martinez and A. Plastino, The role of constraints in Tsallis' nonextensive treatment revisited, Physica A 347, 205 (2005).

[28] S.R.A. Salinas and C. Tsallis, eds., Nonextensive Statistical Mechanics and Thermodynamics, Braz. J. Phys. 29 (1) (1999).

[29] S. Abe and Y. Okamoto, eds., Nonextensive Statistical Mechanics and Its Applications, Series Lecture Notes in Physics (Springer, Berlin, 2001).

[30] P. Grigolini, C. Tsallis and B.J. West, eds., Classical and Quantum Complexity and Nonextensive Thermodynamics, Chaos, Solitons and Fractals 13, Issue 3 (2002).

[31] G. Kaniadakis, M. Lissia and A. Rapisarda, eds., Non Extensive Thermodynamics and Physical Applications, Physica A 305, 1/2 (2002).

[32] M. Sugiyama, ed., Nonadditive Entropy and Nonextensive Statistical Mechanics, Continuum Mechanics and Thermodynamics 16 (Springer-Verlag, Heidelberg, 2004).

[33] H.L. Swinney and C. Tsallis, eds., Anomalous Distributions, Nonlinear Dynamics and Nonextensivity, Physica D 193, Issue 1-4 (2004).

[34] M Gell-Mann and C. Tsallis, eds., Nonextensive Entropy - Interdisciplinary Applications (Oxford University Press, New York, 2004).

[35] G. Kaniadakis and M. Lissia, eds., News and Expectations in Thermostatistics, Physica A 340, Issue 1-3 (2004).

[36] H.J. Herrmann, M. Barbosa and E.M.F. Curado, eds., Trends and Perspectives in Extensive and Non-extensive Statistical Mechanics, Physica A 344, Issue 3/4 (2004).

[37] C. Beck, G. Benedek, A. Rapisarda and C. Tsallis, eds., Complexity, Metastability and Nonextensivity (World Scientific, Singapore, 2005).

[38] J.P. Boon and C. Tsallis, eds., Nonextensive Statistical Mechanics: New Trends, New Perspectives, Europhysics News 36, Number 6, 183-231 (EDP Sciences, Paris, 2005).

[39] G. Kaniadakis, A. Carbone and M. Lissia, eds., News, Expectations and Trends in Statistical Physics, Physica A 365, Issue 1 (2006).

[40] S. Abe, M. Sakagami and N. Suzuki, eds., Complexity and Nonextensivity - New Trends in Statistical Mechanics, Progress of Theoretical Physics Supplement 162 (Physical Society of Japan, 2006).

[41] S. Abe, H. Herrmann, P. Quarati, A. Rapisarda and C. Tsallis, eds., Complexity, Metastability and Nonextensivity, American Institute of Physics Conference Proceedings 965 (New York, 2007).

[42] C. Tsallis, C. Anteneodo, L. Borland and R. Osorio, Nonextensive statistical mechanics and economics, Physica A 324 89 (2003).

[43] M. Bologna, C. Tsallis and P. Grigolini, Anomalous diffusion associated with nonlinear fractional derivative FokkerPlanck-like equation: Exact time-dependent solutions, Phys. Rev. E 62, 2213 (2000)

[44] F.D. Nobre and C. Tsallis, Infinite-range Ising ferromagnet -
Thermodynamic limit within generalized statistical mechanics, Physica A 213, 337 (1995).

[45] C. Anteneodo and C. Tsallis, Multiplicative noise: A mechanism leading to nonextensive statistical mechanics, J. Math. Phys. 44, 5194 (2003).

[46] F.A. Tamarit, S.A. Cannas and C. Tsallis, Sensitivity to initial conditions in the Bak-Sneppen model of biological evolution, Eur. Phys. J. B 1, 545 (1998).

[47] C. Anteneodo and C. Tsallis, Two-dimensional turbulence in pure-electron plasma: A nonextensive thermostatistical description, J. Mol. Liq. 71, 255 (1997).

[48] L. Nivanen, A. Le Mehaute and Q.A. Wang, Generalized algebra within a nonextensive statistics, Rep. Math. Phys. 52, 437 (2003).

[49] E.P. Borges, A possible deformed algebra and calculus inspired in nonextensive thermostatistics, Physica A 340, 95 (2004).

[50] A.R. Plastino and A. Plastino, Non-extensive statistical mechanics and generalized Fokker-Planck equation, Physica A 222, 347 (1995).

[51] C. Tsallis and D.J. Bukman, Anomalous diffusion in the presence of external forces: exact time-dependent solutions and their thermostatistical basis, Phys. Rev. E 54, R2197 (1996).

[52] M.A. Fuentes and M.O. Caceres, Computing the non-linear anomalous diffusion equation from first principles, Phys. Lett. A 372, 1236 (2008).

[53] L. Borland, Ito-Langevin equations within generalized thermostatistics, Phys. Lett. A 245, 67 (1998).

[54] A.M. Mariz and C. Tsallis, (2009) in progress.

[55] W. Braun and K. Hepp, The Vlasov dynamics and its fluctuations in the $1 / N$ limit of interacting classical particles, Comm. Math. Phys. 56, 125 (1977). The reader should be aware that the domain of applicability of this theorem might, in spite of its declared hypothesis, not cover the case of Hamiltonians including long-range interactions at collective states such as the so-called QSS (quasi-stationary state). The 20 March 2006, in a telephone conversation with Prof. Werner Braun (while he was in Texas, and myself at the Santa Fe Institute, New Mexico), he shared with me [nearly literal transcription]: "We were thinking of a potential that has no singularities at the origin and elsewhere, and that decays sufficiently quickly at infinity... In this case we are sure that we are allowed to do all the derivatives and integrals that we do in the paper... Although I cannot be sure after so many years, my guess would be that our arguments are NOT valid if $\alpha<d$. In such a case, I would guess that strange structures could be present in phase space... I am not sure about all this, but this would be my guess. This conversation followed after the e-mail that, in 4 December 2005, I sent to both Prof. Braun and Prof. Hepp. The content of that e-mail (to which I received no reply) was: Dear colleagues, May I ask you a question about your theorem concerning the Vlasov equation? It plays some important role in one of the paradigmatic systems addressed by nonextensive statistical mechanics (see, for instance, http://tsallis.cat.cbpf.br/biblio.htm and also the attachment of the next e-mail, to be published momentarily by the European Physical Society). It is clear for me that the hypothesis assumed in that theorem are fulfilled for Hamiltonian systems that tend to occupy ALL of the associated Gibbs $\Gamma$ phase space. It is intuitively less clear for me that they are fulfilled if the dynamical occupancy in phase space is hierarchical, multifractal-like, possibly with ZERO LEBESGUE MEASURE (like the trajectories of the flights of an airline company, based in say Paris, like Air France, or in Houston, like Continental Airlines, etc). In such anomalous case, there are several numerical indications in the literature indicating 
the enormous influence that initial conditions play. I would certainly be pleased to understand better how these facts can be reconciled. Your clarifying comments would be greatly appreciated. Thank you very much. Best regards, Constantino Tsallis (Santa Fe Institute, New Mexico).

[56] D. Broadhurst (1999), http://pi.lacim.uqam.ca/piDATA/feigenbaum.txt

[57] F. Caruso and C. Tsallis, Nonadditive entropy reconciles the area law in quantum systems with classical thermodynamics, Phys. Rev. E 78, 021102 (2008).

[58] C. Tsallis, M. Gell-Mann and Y. Sato, Asymptotically scaleinvariant occupancy of phase space makes the entropy $S_{q}$ extensive, Proc. Natl. Acad. Sc. USA 102, 15377 (2005).

[59] A. Rodriguez, V. Schwammle and C. Tsallis, Strictly and asymptotically scale-invariant probabilistic models of $N$ correlated binary random variables having q-Gaussians as $N \rightarrow$ $\infty$ limiting distributions, JSTAT P09006 (2008).

[60] R. Hanel, S. Thurner and C. Tsallis, Scale-invariant correlated probabilistic model yields q-Gaussians in the thermodynamic limit, preprint (2008).

[61] E. Lutz, Anomalous diffusion and Tsallis statistics in an optical lattice, Phys. Rev. A 67, 051402(R) (2003).

[62] M.O. Caceres, Computing a non-Maxwellian velocity distribution from first principles, Phys. Rev. E 67, 016102 (2003).

[63] G.F. Mazenko, Vortex velocities in the $O(n)$ symmetric timedependent Ginzburg-Landau model, Phys. Rev. Lett. 78, 401 (1997).

[64] H. Qian and G.F. Mazenko, Vortex dynamics in a coarsening two-dimensional XY model, Phys. Rev. E 68, 021109 (2003).

[65] R. Albert and A.-L. Barabasi, Topology of evolving networks: Local events and universality, Phys. Rev. Lett. 85, 5234 (2000).

[66] B.M. Boghosian, P.J. Love, P.V. Coveney, I.V. Karlin, S. Succi and J. Yepez, Galilean-invariant lattice-Boltzmann models with H-theorem, Phys. Rev. E 68, 025103(R) (2003).

[67] B.M. Boghosian, P.J. Love, J. Yepez and P.V. Coveney, Galilean-invariant multi-speed entropic lattice Boltzmann models, in Anomalous Distributions, Nonlinear Dynamics and Nonextensivity, eds. H.L. Swinney and C. Tsallis, Physica D 193, 169 (2004).

[68] B.M. Boghosian, General theory of Galilean-invariant entropic lattice Boltzmann models, in Complexity, Metastability and Nonextensivity, eds. C. Beck, G. Benedek, A. Rapisarda and C. Tsallis (World Scientific, Singapore, 2005), page 185.

[69] A.M. Scarfone, P. Quarati, G. Mezzorani and M. Lissia, Analytical predictions of non-Gaussian distribution parameters for stellar plasmas, Astrophysics and Space Science 315, 353 (2008).

[70] A. Rossani and A.M. Scarfone, Generalized Pearson distributions for charged particles interacting with an electric and/or a magnetic field, Physica A 388, 2354 (2009).

[71] E. Mayoral and A. Robledo, Tsallis' $q$ index and Mori's $q$ phase transitions at edge of chaos, Phys. Rev. E 72, 026209 (2005).

[72] H. Hernandez-Saldana and A. Robledo, Dynamics at the quasiperiodic onset of chaos, Tsallis q-statistics and Mori's q-phase transitions, Physica A 370, 286 (2006).

[73] R.S. Mendes and C. Tsallis, Renormalization group approach to nonextensive statistical mechanics, Phys. Lett. A 285, 273 (2001).

[74] S. Umarov, C. Tsallis, M. Gell-Mann and S. Steinberg, Symmetric $(q, \alpha)$-stable distributions. Part I: First representation, cond-mat/0606038v2 (2008).

[75] S. Umarov, C. Tsallis, M. Gell-Mann and S. Steinberg, Symmetric $(q, \alpha)$-stable distributions. Part I: Second representation, cond-mat/0606040v2 (2008).
[76] K.P. Nelson and S. Umarov, The relationship between Tsallis statistics, the Fourier transform, and nonlinear coupling, preprint (2008), 0811.3777 [cs.IT].

[77] C. Tsallis, Dynamical scenario for nonextensive statitical mechanics, Physica A 340, 1 (2004).

[78] L.F. Burlaga and A.F. Vinas, Triangle for the entropic index $q$ of non-extensive statistical mechanic observed by Voyager 1 in the distant heliosphere, Physica A 356, 375 (2005).

[79] N.O. Baella, private communication (2008).

[80] P. Grassberger, Temporal scaling at Feigenbaum points and nonextensive thermodynamics, Phys. Rev. Lett. 95, 140601 (2005).

[81] C. Tsallis, Comment on "Temporal scaling at Feigenbaum points and nonextensive thermodynamics" by P. Grassberger, cond-mat/0511213 (2005).

[82] A. Robledo, Incidence of nonextensive thermodynamics in temporal scaling at Feigenbaum points, Physica A 370, 449 (2006).

[83] A. Robledo and L.G. Moyano, q-deformed statisticalmechanical property in the dynamics of trajectories en route to the Feigenbaum attractor, Phys. Rev. E 77, 032613 (2008).

[84] U. Tirnakli, C. Beck and C. Tsallis, Central limit behavior of deterministic dynamical systems, Phys. Rev. E 75, 040106(R) (2007).

[85] U. Tirnakli, C. Tsallis and C. Beck, A closer look at time averages of the logistic map at the edge of chaos, Phys. Rev. E 79, 056209 (2009). This paper replies also to criticism by P. Grassberger, Proposed central limit behavior in deterministic dynamical systems, Phys. Rev. E 79, 057201 (2009).

[86] C. Tsallis, Nonextensive statistical mechanics, anomalous diffusion and central limit theorems, Milan Journal of Mathematics 73, 145 (2005).

[87] J.P Bouchaud and A. Georges, Anomalous diffusion in disordered media: Statistical mechanisms, models and physical applications, Phys. Rep. 195, 127 (1990).

[88] C. Tsallis and S.M.D. Queiros, Nonextensive statistical mechanics and central limit theorems I - Convolution of independent random variables and q-product, in Complexity, Metastability and Nonextensivity, eds. S. Abe, H.J. Herrmann, P. Quarati, A. Rapisarda and C. Tsallis, American Institute of Physics Conference Proceedings 965, 8 (New York, 2007).

[89] S.M.D. Queiros and C. Tsallis, Nonextensive statistical mechanics and central limit theorems II - Convolution of $q$ independent random variables, in Complexity, Metastability and Nonextensivity, eds. S. Abe, H.J. Herrmann, P. Quarati, A. Rapisarda and C. Tsallis, American Institute of Physics Conference Proceedings 965, 21 (New York, 2007).

[90] H.J. Hilhorst (2008), private communication.

[91] H.J. Hilhorst, Braz. J. Phys. (2009), in the present volume.

[92] C. Tsallis and E.M.F. Curado, unpublished.

[93] C. Vignat and A. Plastino, Central limit theorem and deformed exponentials, J. Phys. A 40, F969 (2007).

[94] I. Podlubny, Fractional Differential Equations (Academic Press, San Diego, 1999).

[95] R. Metzler and J. Klafter, The random walk's guide to anomalous diffusion: A fractional dynamics approach, Phys. Rep. 339, 1 (2000).

[96] R. Hilfer, ed., Applications Of Fractional Calculus In Physics (World Scientific, Singapore, 2000)

[97] P. Douglas, S. Bergamini and F. Renzoni, Tunable Tsallis distributions in dissipative optical lattices, Phys. Rev. Lett. 96, 110601 (2006)

[98] B. Liu and J. Goree, Superdiffusion and non-Gaussian statistics in a driven-dissipative $2 D$ dusty plasma, Phys. Rev. Lett. 100, 055003 (2008)

[99] B. Liu, J. Goree and Y. Feng, Non-Gaussian statistics and su- 
perdiffusion in a driven-dissipative dusty plasma, Phys. Rev. E 78, 046403 (2008).

[100] R.G. DeVoe, Power-law distributions for a trapped ion interacting with a classical buffer gas, Phys. Rev. Lett. 102, 063001 (2009).

[101] A. Upadhyaya, J.-P. Rieu, J.A. Glazier and Y. Sawada, Anomalous diffusion and non-Gaussian velocity distribution of Hydra cells in cellular aggregates, Physica A 293, 549 (2001).

[102] K.E. Daniels, C. Beck and E. Bodenschatz, Defect turbulence and generalized statistical mechanics, Physica D 193, 208 (2004).

[103] L.F. Burlaga, N.F. Ness and M.H. Acuna, Multiscale structure of magnetic fields in the heliosheath, J. Geophysical Res.Space Physics 111, A09112 (2006).

[104] L.F. Burlaga, A.F. Vinas, N.F. Ness and M.H. Acuna, Tsallis statistics of the magnetic field in the heliosheath, Astrophys. J. 644, L83 (2006).

[105] L.F. Burlaga, A. F-Vinas and C. Wang, Tsallis distributions of magnetic field strength variations in the heliosphere: 5 to 90 $A U$, J. Geophysical Res.-Space Physics 112, A07206 (2007).

[106] L.F. Burlaga, N.F. Ness, M.H. Acuna, Magnetic fields in the heliosheath and distant heliosphere: Voyager 1 and $2 \mathrm{ob}$ servations during 2005 and 2006, Astrophys. J. 668, 1246 (2007).

[107] L.F. Burlaga, N.F. Ness and M.H. Acuna, Magnetic field strength fluctuations and temperature in the heliosheath, Astrophys. J. 691, L82 (2009).

[108] R. Arevalo, A. Garcimartin and D. Maza, Anomalous diffusion in silo drainage, Eur. Phys. J. E 23, 191 (2007).

[109] R. Arevalo, A. Garcimartin and D. Maza, A non-standard statistical approach to the silo discharge, in Complex Systems New Trends and Expectations, eds. H.S. Wio, M.A. Rodriguez and L. Pesquera, Eur. Phys. J.-Special Topics 143 (2007).

[110] A. Pluchino, A. Rapisarda and C. Tsallis, Nonergodicity and central limit behavior in long-range Hamiltonians, Europhys. Lett. 80, 26002 (2007).

[111] A. Pluchino and A. Rapisarda, Nonergodicity and central limit behavior for systems with long-range interactions, SPIE 2, 6802-32 (2007)

[112] A. Pluchino, A. Rapisarda and C. Tsallis, A closer look at the indications of q-generalized Central Limit Theorem behavior in quasi-stationary states of the HMF model, Physica A 387, 3121 (2008). For criticism and its rebuttal see A. Figueiredo, T.M. Rocha Filho and M.A. Amato, Ergodicity and central limit theorem in systems with long-range interactions, Europhys. Lett. 83, 30011 (2008); A. Pluchino, A. Rapisarda and C. Tsallis, Comment on "Ergodicity and central limit theorem in systems with long-range interactions" by Figueiredo A. et $a l$, Europhys. Lett. 85, 60006 (2009); A. Figueiredo, T.M. Rocha Filho and M.A. Amato, Reply to to the Comment by A. Pluchino et al, Europhys. Lett. 85, 60007 (2009).

[113] T. Dauxois, S. Ruffo, E. Arimondo and M. Wilkens, eds., $D y-$ namics and Thermodynamics of Systems with Long Range Interactions, Lecture Notes in Physics 602 (Springer, Berlin, 2002).

[114] A. Antoniazzi, D. Fanelli, J. Barre, P.-H. Chavanis, T. Dauxois and S. Ruffo, Maximum entropy principle explains quasistationary states in systems with long-range interactions: The example of the Hamiltonian mean-field model, Phys. Rev. E
75, 011112 (2007).

[115] G. Miritello, A. Pluchino and A. Rapisarda, Central limit violation in the Kuramoto model at the 'edge of chaos', preprint (2009), 0903.4400 [cond-mat.stat-mech].

[116] G. Ruiz and C. Tsallis, Nonextensivity at the edge of chaos of a new universality class of one-dimensional unimodal dissipative maps, Eur. Phys. J. B 67, 577 (2009).

[117] F. Caruso, A. Pluchino, V. Latora, S. Vinciguerra and A. Rapisarda, Analysis of self-organized criticality in the OlamiFeder-Christensen model and in real earthquakes, Phys. Rev. E 75, 055101(R)(2007).

[118] B. Bakar and U. Tirnakli, Analysis of self-organized criticality in Ehrenfest's dog-flea model, Phys. Rev. E 79, 040103 (2009).

[119] M. Suvakov and B. Tadic, Collective charge fluctuations in single-electron processes on nanonetworks, J. Stat. Mech. P02015 (2009).

[120] L.Borland, Option pricing formulas based on a non-Gaussian stock price model, Phys. Rev. Lett. 89, 098701 (2002).

[121] L. Borland, A theory of non-gaussian option pricing, Quantitative Finance 2, 415 (2002).

[122] C. Tsallis, C. Anteneodo, L. Borland and R. Osorio, Nonextensive statistical mechanics and economics, Physica A 324, 89 (2003).

[123] R. Osorio, L. Borland and C. Tsallis, Distributions of highfrequency stock-market observables, in Nonextensive Entropy - Interdisciplinary Applications, eds. M. Gell-Mann and C. Tsallis (Oxford University Press, New York, 2004).

[124] L. Borland and J.-P. Bouchaud, A non-Gaussian option pricing model with skew, Quant. Finance 4, 499 (2004).

[125] L. Borland, The pricing of stock options, in Nonextensive Entropy - Interdisciplinary Applications, eds. M. Gell-Mann and C. Tsallis (Oxford University Press, New York, 2004).

[126] L. Borland, Long-range memory and nonextensivity in financial markets, in Nonextensive Statistical Mechanics: New Trends, New perspectives, eds. J.P. Boon and C. Tsallis, Europhysics News 36, 228 (2005).

[127] L. Borland, A non-Gaussian model of stock returns: option smiles, credit skews, and a multi-time scale memory, in Noise and Fluctuations in Econophysics and Finance, eds. D. Abbott, J.-P. Bouchaud, X. Gabaix and J.L. McCauley, Proc. of SPIE 5848, 55 (SPIE, Bellingham, WA, 2005).

[128] S.M.D. Queiros, On non-Gaussianity and dependence in $\mathrm{fi-}$ nancial in time series: A nonextensive approach, Quant. Finance 5, 475 (2005)

[129] L. Borland, A non-Gaussian stock price model: Options, credit and a multi-timescale memory, in Complexity and Nonextensivity: New Trends in Statistical Mechanics, eds. M. Sakagami, N. Suzuki and S. Abe, Prog. Theor. Phys. Suppl. 162, 155 (2006).

[130] A.A.G. Cortines and R. Riera Freire, Non-extensive behavior of a stock market index at microscopic time scales, Physica A 377, 181 (2007).

[131] T.D. Frank, Nonextensive cutoff distributions of postural sway for the old and the young, Physica A 388, 2503 (2009).

[132] R.M. Pickup, R. Cywinski, C. Pappas, B. Farago and P. Fouquet, Generalized spin glass relaxation, Phys. Rev. Lett. 102, 097202 (2009). 